\title{
BOUNDS FOR TRACES OF HECKE OPERATORS AND APPLICATIONS TO MODULAR AND ELLIPTIC CURVES OVER A FINITE FIELD
}

\author{
IAN PETROW
}

\begin{abstract}
We give an upper bound for the trace of a Hecke operator acting on the space of holomorphic cusp forms with respect to certain congruence subgroups. Such an estimate has applications to the analytic theory of elliptic curves over a finite field, going beyond the Riemann hypothesis over finite fields. As the main tool to prove our bound on traces of Hecke operators, we develop a Petersson formula for newforms for general nebentype characters.
\end{abstract}

\section{INTRODUCTION}

1.1. Statement of Results. Let $S_{\kappa}(\Gamma, \epsilon)$ be the space of holomorphic cusp forms of weight $\kappa$, for a subgroup $\Gamma$ of a Hecke congruence group, and of nebentype character $\epsilon$. We write $\operatorname{Tr}\left(T \mid S_{\kappa}(\Gamma, \epsilon)\right)$ for the trace of a linear operator $T$ acting on $S_{\kappa}(\Gamma, \epsilon)$. The aim of this paper is to give estimates for $\operatorname{Tr}\left(T_{m} \mid S_{\kappa}(\Gamma, \epsilon)\right)$, where $T_{m}$ is the $m$ th Hecke operator, as the parameters $m, \kappa, \Gamma$, and $\epsilon$ vary simultaneously.

Consider first the case that $\Gamma=\Gamma_{0}(N)$ and $\epsilon$ is any Dirichlet character modulo $N$. Let $d(m)$ denote the number of divisors of $m, \sigma(m)$ the sum of the divisors of $m$, and let $\psi(N)=\left[\Gamma_{0}(N)\right.$ : $\left.\mathrm{SL}_{2}(\mathbf{Z})\right]=N \prod_{p \mid N}\left(1+\frac{1}{p}\right)$. We assume that $\kappa \geqslant 2$ an integer throughout the paper. Deligne's theorem tells us that each eigenvalue $\lambda(m)$ of $T_{m}$ satisfies $|\lambda(m)| \leqslant d(m) m^{\frac{\kappa-1}{2}}$. Therefore we have the "trivial" estimate on the trace

$$
\operatorname{Tr}\left(T_{m} \mid S_{\kappa}\left(\Gamma_{0}(N), \epsilon\right)\right) \leqslant \operatorname{dim} S_{\kappa}\left(\Gamma_{0}(N), \epsilon\right) d(m) m^{\frac{\kappa-1}{2}} \leqslant \frac{(\kappa-1) \psi(N)}{12} d(m) m^{\frac{\kappa-1}{2}} .
$$

For the bound on $\operatorname{dim} S_{\kappa}\left(\Gamma_{0}(N), \epsilon\right)$, see e.g. [Ros92, Cor 8]. The power of $m$ in (1.1) is sharp by the Sato-Tate distribution for Hecke eigenvalues. On the other hand, by a careful analysis using the Eichler-Selberg trace formula, Conrey, Duke and Farmer [CDF97] and in more generality Serre [Ser97, Prop. 4] show that if $\epsilon(-1)=(-1)^{\kappa}$ then

$$
\operatorname{Tr}\left(T_{m} \mid S_{\kappa}\left(\Gamma_{0}(N), \epsilon\right)\right)=\frac{\kappa-1}{12} \epsilon\left(m^{\frac{1}{2}}\right) m^{\frac{\kappa}{2}-1} \psi(N)+O\left(\left(\sigma(m) \max _{f^{2}<4 m} \psi(f)+d(m) N^{\frac{1}{2}}\right) m^{\frac{\kappa-1}{2}} d(N)\right),
$$

where $\epsilon\left(m^{1 / 2}\right)$ is understood to be 0 unless $m$ is a perfect square. If $\epsilon(-1) \neq(-1)^{\kappa}$ then $S_{\kappa}\left(\Gamma_{0}(N), \epsilon\right)=$ $\{0\}$, so the left hand side vanishes identically. We expect the estimate (1.2) to be sharp if $m$ is fixed and $\kappa+N \rightarrow \infty$.

Write $\mathbf{c}(\epsilon)$ for the conductor of the Dirichlet character $\epsilon$, and $\mathbf{c}^{*}(\epsilon)=\prod_{p \mid \mathbf{c}(\epsilon)} p$ for its square-free part. In this paper we prove:

Theorem 1.1. Suppose that $\epsilon(-1)=(-1)^{\kappa},(N, m)=1$, and that $m \mathbf{c}(\epsilon) \mathbf{c}^{*}(\epsilon) \ll\left(N^{4} \kappa^{10 / 3}\right)^{1-\eta}$ for some $\eta>0$. Then we have that

$$
\operatorname{Tr}\left(T_{m} \mid S_{\kappa}\left(\Gamma_{0}(N), \epsilon\right)\right)=\frac{\kappa-1}{12} \epsilon\left(m^{\frac{1}{2}}\right) m^{\frac{\kappa}{2}-1} \psi(N)+O_{\eta, \varepsilon}\left(N^{\frac{10}{11}} m^{\frac{\kappa-1}{2}+\frac{1}{44}} \kappa^{\frac{61}{66}} \mathbf{c}(\epsilon)^{\frac{1}{44}} \mathbf{c}^{*}(\epsilon)^{\frac{1}{44}}(N m \kappa)^{\varepsilon}\right) .
$$

This work was supported by Swiss National Science Foundation grant PZ00P2_168164. 
We remark that the hypothesis that $m \mathbf{c}(\epsilon) \mathbf{c}^{*}(\epsilon) \ll\left(N^{4} \kappa^{10 / 3}\right)^{1-\eta}$ for some $\eta>0$ in Theorem 1.1 is no restriction in practice, since if the hypothesis fails then (1.1) is a superior bound anyway. Indeed, the error term in (1.3) is smaller than that in both (1.1) and (1.2) when

$$
N^{\frac{8}{13}} \kappa^{\frac{122}{195}}(N \kappa)^{\varepsilon} \mathbf{c}(\epsilon)^{\frac{1}{65}} \mathbf{c}^{*}(\epsilon)^{\frac{1}{65}} \ll m \ll \frac{\left(N^{4} \kappa^{\frac{10}{3}}\right)^{1-\eta}}{\mathbf{c}(\epsilon) \mathbf{c}^{*}(\epsilon)} .
$$

For example, if $\epsilon$ is trivial and the weight $\kappa$ is fixed, then (1.3) is better than (1.1) and (1.2) for

$$
N^{\frac{8}{13}+\varepsilon} \ll m \ll N^{4-\varepsilon} .
$$

Note that our result requires the hypothesis $(N, m)=1$, whereas the estimates (1.1) and (1.2) do not. We discuss the source of this condition in the sketch of the proof, below.

We are also interested in spaces of modular forms for groups other than $\Gamma_{0}(N)$. In particular, for positive integers $M \mid N$ let

$$
\Gamma(M, N)=\left\{\left(\begin{array}{ll}
a & b \\
c & d
\end{array}\right) \in \mathrm{SL}_{2}(\mathbf{Z}) \text { s.t. } a, d \equiv 1(\bmod N), c \equiv 0(\bmod N M)\right\} .
$$

These congruence groups interpolate between $\Gamma_{1}(N)=\Gamma(1, N)$ and $\Gamma(N) \simeq \Gamma(N, N)$. We write $S_{\kappa}(M, N)$ for the space of modular forms of weight $\kappa$ for the group $\Gamma(M, N)$ (without nebentype character). Let $\delta(a, b)$ be the indicator function of $a=b$ and $\delta_{c}(a, b)$ be the indicator function of $a \equiv b(\bmod c)$. Let $T_{m}$ be the $m$ th Hecke operator acting on $S_{\kappa}(M, N)$ and for $(d, N)=1$ let $\langle d\rangle$ the $d$ th diamond operator. These operators commute and $T_{1}=\langle 1\rangle=\mathrm{id}$; for definitions see DS05, $\S 5.1,5.2]$ or [KP17, §4]. In particular, we have

$$
\operatorname{Tr}\left(\langle d\rangle T_{m} \mid S_{\kappa}(\Gamma(M, N))\right)=\sum_{\epsilon(\bmod N)} \epsilon(d) \operatorname{Tr}\left(T_{m} \mid S_{\kappa}\left(\Gamma_{0}(N M), \epsilon\right)\right) .
$$

Applying (1.1) to (1.5) we have

$$
\operatorname{Tr}\left(\langle d\rangle T_{m} \mid S_{\kappa}(\Gamma(M, N))\right) \leqslant \frac{\kappa-1}{12} \varphi(N) \psi(N M) d(m) m^{\frac{\kappa-1}{2}} .
$$

Meanwhile, summing (1.2) over characters $\epsilon(\bmod N)$ such that $\epsilon(-1)=(-1)^{\kappa}$ we find

$$
\begin{array}{r}
\operatorname{Tr}\left(\langle d\rangle T_{m} \mid S_{\kappa}(\Gamma(M, N))\right)=\frac{\kappa-1}{24} m^{\frac{\kappa}{2}-1} \varphi(N) \psi(N M)\left(\delta_{N}\left(m^{\frac{1}{2}} d, 1\right)+(-1)^{\kappa} \delta_{N}\left(m^{\frac{1}{2}} d,-1\right)\right) \\
+O\left(\left(\sigma(m) \max _{f^{2}<4 m} \psi(f)+d(m)(M N)^{\frac{1}{2}}\right) m^{\frac{\kappa-1}{2}} d(M N) N\right) .
\end{array}
$$

The following result improves on both (1.6) and (1.7) in an intermediate range of parameters.

Theorem 1.2. Suppose that $M \mid N,(N, m)=1$, and that $m \ll\left(N^{6} \kappa^{10 / 3}\right)^{1-\eta}$ for some $\eta>0$. We have that

$$
\begin{aligned}
\operatorname{Tr}\left(\langle d\rangle T_{m} \mid S_{\kappa}(\Gamma(M, N))\right)=\frac{\kappa-1}{24} m^{\frac{\kappa}{2}-1} \varphi(N) \psi(N M)\left(\delta_{N}\left(m^{\frac{1}{2}} d, 1\right)+(-1)^{\kappa} \delta_{N}\left(m^{\frac{1}{2}} d,-1\right)\right) \\
+O_{\eta, \varepsilon}\left(M N^{\frac{41}{22}} m^{\frac{\kappa-1}{2}+\frac{1}{44}} \kappa^{\frac{61}{66}}(N m \kappa)^{\varepsilon}\right) .
\end{aligned}
$$

1.2. Applications to Modular and Elliptic Curves over a Finite Field. Hecke operators appear throughout number theory, and estimates for their traces are especially relevant to equidistribution problems. See for example [Ser97, §5- $\S 8]$ and [MS09]. We mention here a few consequences in the analytic theory of modular and elliptic curves over a finite field.

Let $C$ be a nonsingular projective curve of genus $g$ over a finite field $\mathbf{F}_{q}$ with $q$ elements. Then we have (see e.g. [Mil17, Ch. 11]) that

$$
\left|C\left(\mathbf{F}_{q^{n}}\right)\right|=q^{n}+1-\sum_{i=1}^{2 g} \alpha_{i}^{n},
$$


where $\left\{\alpha_{i}\right\}$ are the inverse zeros of the zeta function of $C$

$$
Z(C, T)=\frac{\left(1-\alpha_{1} T\right) \cdots\left(1-\alpha_{2 g} T\right)}{(1-T)(1-q T)} .
$$

The Riemann hypothesis for curves over finite fields asserts that $\left|\alpha_{i}\right|=\sqrt{q}$ for all $i$. Igusa Igu59 showed that there exists a non-singular projective model for $X_{0}(N)$ over $\mathbf{Q}$ whose reductions modulo primes $p, p \nmid N$ are also non-singular (see also the survey [DI95, §9]), and so the preceding discussion applies to $X_{0}(N)$ when $p \nmid N$. Since $g \sim \psi(N) / 12$ as $N \rightarrow \infty$ we have that

$$
\left|X_{0}(N)\left(\mathbf{F}_{q}\right)\right|=q+1+O\left(\psi(N) q^{1 / 2}\right) .
$$

In particular, $\left|X_{0}(N)\left(\mathbf{F}_{q}\right)\right| \sim q$ as $q \rightarrow \infty$ as soon as $q \gg N^{2+\delta}$ for some $\delta>0$. On the other hand, the Eichler-Shimura correspondence (see e.g. [Mil17, Thm. 11.14]) asserts that

$$
Z\left(X_{0}(N), T\right)=\frac{\prod_{f \in H_{2}(N)}\left(1-\lambda_{f}(p) T+p T^{2}\right)}{(1-T)(1-p T)},
$$

where $H_{2}(N)$ is a basis for $S_{2}\left(\Gamma_{0}(N)\right)$ consisting of eigenforms of $\left\{T_{p}, p \nmid N\right\}$, and $\lambda_{f}(p)$ is the $T_{p}$ eigenvalue of $f$. We therefore have

$$
\left|X_{0}(N)\left(\mathbf{F}_{q}\right)\right|=q+1-\operatorname{Tr}\left(T_{q} \mid S_{2}\left(\Gamma_{0}(N)\right)\right)+p \operatorname{Tr}\left(T_{q / p^{2}} \mid S_{2}\left(\Gamma_{0}(N)\right)\right),
$$

where we set $T_{p^{-1}}=0$. Applying (1.1), (1.2), and Theorem 1.1 we get

Corollary 1.3. Suppose $q=p^{v}$ is a prime power such that $p \nmid N$. We have

$$
\left|X_{0}(N)\left(\mathbf{F}_{q}\right)\right|=q+(p-1) \frac{\psi(N)}{12} \delta_{2}(v, 0)+O_{\varepsilon}\left(\min \left(\psi(N), q^{\frac{1}{44}} N^{\frac{10}{11}}(q N)^{\varepsilon},\left(q^{\frac{3}{2}}+N^{\frac{1}{2}}\right) d(N) q^{\varepsilon}\right) q^{\frac{1}{2}}\right) .
$$

In particular, the main term is larger than the error term as soon as $q \gg N^{\frac{40}{21}+\delta}$ for some fixed $\delta>0$.

Corollary 1.3 shows that there is significant cancellation between the zeros $\alpha_{i}$ of $Z\left(X_{0}(N), T\right)$, and in this sense goes beyond the Riemann hypothesis for $Z\left(X_{0}(N), T\right)$. Assuming square-root cancellation between the zeros, one might conjecture an error term of size $(q N)^{1 / 2+\varepsilon}$ in Corollary 1.3. which would imply that the main term is larger than the error term whenever $q \gg N^{1+\delta}$ for some $\delta$. If one assumes the generalized Lindelöf hypothesis for adjoint square $L$-functions, then the method in this paper produces an error term of size $q^{1 / 8+\varepsilon} N^{1 / 2+\varepsilon}$ in Corollary 1.3 (see Lemma 6.1). In a much more speculative direction, if under the assumption $(m n, W)=1$ the upper bound $\ll_{\kappa, \varepsilon}(m n W)^{\varepsilon} W^{-1 / 2}$ for the sum appearing in Lemma 4.1 holds (cf. the Linnik-Selberg conjecture), then the error term $(q N)^{1 / 2+\varepsilon}$ in Corollary 1.3 is admissible.

If $q$ is a square then we can compare the second main term in Corollary 1.3 to the error term coming from (1.2) in the range where $q$ is small compared to $N$. For example, in the special case that $p$ is a prime and $q=p^{2}$ we have

Corollary 1.4. If $p, N \rightarrow \infty$ where $p$ runs through primes $p \nmid N$ then for any fixed $\delta>0$ we have

$$
\left|X_{0}(N)\left(\mathbf{F}_{p^{2}}\right)\right|= \begin{cases}p^{2}+O(p \psi(N)) & \text { if } p^{2} \gg N^{4-\delta} \\ p^{2}+p \frac{\psi(N)}{12}+O_{\varepsilon}\left(p^{\frac{23}{22}} N \frac{10}{11}^{12}(q N)^{\varepsilon}\right) & \text { if } N^{\frac{40}{21}-\delta} \ll p^{2} \ll N^{4-\delta} \\ p \frac{\psi(N)}{12}+O_{\varepsilon}\left(p^{\frac{23}{22}} N^{\frac{10}{11}}(q N)^{\varepsilon}\right) & \text { if } N^{\frac{8}{13}}+\delta \ll p^{2} \ll N^{\frac{40}{21}-\delta} \\ (p-1) \frac{\psi(N)}{12}+O_{\varepsilon}\left(\left(p^{4}+N^{\frac{1}{2}} p\right) d(N) p^{\varepsilon}\right) & \text { if } p^{2} \ll N^{\frac{2}{3}-\delta} .\end{cases}
$$

The first of these cases is just (1.8), and the last is the Tsfasman-Vlăduţ-Zink theorem [TVtZ82, which has important applications to algebraic coding theory, see [Mor91, Ch. 5].

Using Theorem 1.2 we can make more explicit statements about elliptic curves themselves. Let $E$ be an elliptic curve defined over $\mathbf{F}_{q}$ and let $t_{E}=q+1-\# E\left(\mathbf{F}_{q}\right)$ be the trace of the associated 
Frobenius endomorphism. Hasse's Theorem tells us that $\left|t_{E}\right| \leqslant 2 \sqrt{q}$. The set of $\mathbf{F}_{q}$-isomorphism classes of elliptic curves defined over $\mathbf{F}_{q}$ is naturally a probability space where the probability of a singleton is given by

$$
\mathbf{P}_{q}(\{E\})=\frac{1}{q\left|\operatorname{Aut}_{\mathbf{F}_{q}}(E)\right|} .
$$

We would like to study the expectations as $q \rightarrow \infty$ of various random variables associated to $t_{E}$ or the structure of the group of $\mathbf{F}_{q}$-rational points of $E$. To be precise: let $A$ be a finite abelian group with at most two generators, and let $\Phi_{A}$ denote the indicator function of the event that there exists an injective group homomorphism $A \hookrightarrow E\left(\mathbf{F}_{q}\right)$. Let $U_{j}(x)$ for $j \geqslant 0$ be the Chebyshev polynomials of the second kind. The Chebyshev polynomials form an orthonormal basis for the Hilbert space $L^{2}\left([-1,1], \frac{2}{\pi} \sqrt{1-x^{2}} d x\right)$. N. Kaplan and the author in [KP17, Thm. 2] gave explicit formulas for the expectations

$$
\mathbf{E}_{q}\left(U_{j}\left(t_{E} / 2 \sqrt{q}\right) \Phi_{A}\right)=\frac{1}{q} \sum_{\substack{E / \mathbf{F}_{q} \\ A \hookrightarrow E\left(\mathbf{F}_{q}\right)}} \frac{U_{j}\left(t_{E} / 2 \sqrt{q}\right)}{\left|\operatorname{Aut}_{\mathbf{F}_{q}}(E)\right|}
$$

in terms of $\operatorname{Tr}\left(\langle d\rangle T_{m} \mid S_{\kappa}(\Gamma(M, N))\right)$ and elementary arithmetic functions of $m, M, N$, and $j$.

Theorem 1.2 yields the following refinement of the error term in the main corollary of [KP17]. Let

$$
v\left(n_{1}, n_{2}\right)=\frac{n_{1}}{\psi\left(n_{1}\right) \varphi\left(n_{1}\right) n_{2}^{2}} \prod_{\ell \mid \frac{n_{1}}{\left(q-1, n_{1}\right)}}\left(1+\ell^{-1-2 v_{\ell}\left(\frac{\left(q-1, n_{1}\right)}{n_{2}}\right)}\right) .
$$

Corollary 1.5. Let $n_{1}=n_{1}(A)$ and $n_{2}=n_{2}(A)$ be the first and second invariant factors of $A$ (i.e. we have $\left.n_{2} \mid n_{1}\right)$. Suppose that $(|A|, q)=1$ and $q \equiv 1\left(\bmod n_{2}\right)$. Then

$$
\mathbf{E}_{q}\left(U_{j}\left(t_{E} / 2 \sqrt{q}\right) \Phi_{A}\right)=v\left(n_{1}, n_{2}\right)\left(\delta(j, 0)+O_{j, \varepsilon}\left(\min \left(n_{1}, q^{\frac{1}{44}} n_{1}^{\frac{19}{22}}\right) n_{1} n_{2} q^{-\frac{1}{2}}\left(q n_{1}\right)^{\varepsilon}\right)\right) .
$$

If $q \not \equiv 1\left(\bmod n_{2}\right)$, then $\mathbf{E}_{q}\left(U_{j} \Phi_{A}\right)$ vanishes identically.

In particular, the traces of the Frobenius $t_{E}$ for $\left\{E / \mathbf{F}_{q}: A \hookrightarrow E\left(\mathbf{F}_{q}\right)\right\}$ become equidistributed with respect to the Sato-Tate measure as $q \rightarrow \infty$ through prime powers $q \equiv 1\left(\bmod n_{2}\right)$. The equidistribution is uniform in $A$ as soon as $q \gg n_{2}^{2} n_{1}^{\frac{41}{11}+\delta}$ for any fixed $\delta>0$.

In [KP17] Kaplan and the author showed that the equidistribution of $t_{E}$ for $\left\{E / \mathbf{F}_{q}: A \hookrightarrow E\left(\mathbf{F}_{q}\right)\right\}$ is uniform as soon as $q \gg n_{2}^{2} n_{1}^{4+\delta}$ by applying (1.6) to bound the trace. In this sense, Corollary 1.5 goes beyond what one can conclude using the Riemann hypothesis of Deligne alone. All of the error terms in the theorems and corollaries found in section 2 of [KP17] are similarly improved by applying Theorem 1.2 in addition to (1.6).

1.3. Outline of Proof. Thanks to (1.5), the structural steps of the proof of Theorem 1.2 reduce to those of Theorem 1.1. The details of the analytic arguments differ however (see section 5). For these reasons, we only discuss the proof of Theorem 1.1 in this outline.

By Atkin-Lehner theory, to estimate $\operatorname{Tr}\left(T_{m} \mid S_{\kappa}\left(\Gamma_{0}(N), \epsilon\right)\right)$ it suffices to estimate

$$
\sum_{f \in H_{\kappa}^{\star}(N, \epsilon)} \lambda_{f}(m)
$$

where $H_{\kappa}^{\star}(N, \epsilon)$ is set of Hecke-normalized newforms of level $N$ and character $\epsilon$, and $\lambda_{f}(m)$ is the $m$ th Hecke eigenvalue of $f$, normalized so that $\left|\lambda_{f}(n)\right| \leqslant d(n)$. Whereas Serre and Conrey, Duke, and Farmer used the Eichler-Selberg trace formula to access the trace of $T_{m}$, we take a different path and use the Petersson trace formula. 
Let $\mathcal{B}_{\kappa}\left(\Gamma_{0}(N), \epsilon\right)$ be an orthonormal basis for $S_{\kappa}\left(\Gamma_{0}(N), \epsilon\right)$. Let $g \in \mathcal{B}_{\kappa}\left(\Gamma_{0}(N), \epsilon\right)$ and write its Fourier coefficients as $\left\{b_{g}(n)\right\}_{n \geqslant 1}$. Then the Petersson formula [IK04, Prop. 14.5] says that (1.10)

$$
\frac{\Gamma(\kappa-1)}{(4 \pi \sqrt{m n})^{\kappa-1}} \sum_{f \in \mathcal{B}_{\kappa}\left(\Gamma_{0}(N), \epsilon\right)} b_{f}(n) \overline{b_{f}(m)}=\delta(m, n)+2 \pi i^{-\kappa} \sum_{\substack{c>0 \\ c \equiv 0(\bmod N)}} \frac{S_{\epsilon}(m, n, c)}{c} J_{\kappa-1}\left(\frac{4 \pi \sqrt{m n}}{c}\right),
$$

where $J_{\alpha}$ is the $J$-Bessel function, $S_{\epsilon}(m, n, c)$ is the twisted Kloosterman sum

$$
S_{\epsilon}(m, n, c)=\sum_{d(\bmod c)}^{*} \epsilon(d) e\left(\frac{d m+\bar{d} n}{c}\right)
$$

and the $*$ indicates we run over invertible $d(\bmod c)$.

Our goal is to apply the Petersson formula to (1.9), and so we are faced with two technical difficulties:

(1) Only the newforms in $S_{\kappa}\left(\Gamma_{0}(N), \epsilon\right)$ have Fourier coefficients proportional to the Hecke eigenvalues appearing in (1.9), and

(2) If $f$ is a newform, the constant of proportionality between Fourier coefficients $b_{f}(n)$ and the Hecke eigenvalues $\lambda_{f}(n)$ is $\approx\|f\|_{L^{2}}$, which is not constant across $H_{\kappa}^{\star}(N, \epsilon)$.

We overcome (1) in Theorem 3.1 by developing a Petersson formula for newforms for $S_{\kappa}\left(\Gamma_{0}(N), \epsilon\right)$. There has been much recent interest in such formulas, see for example [BBD ${ }^{+}$17, [Nel17, [PY18, and You17. Theorem 3.1 is a generalization of [BBD ${ }^{+17}$, Prop. 4.1] to nontrivial central characters, which itself is a generalization of work of Iwaniec-Luo-Sarnak [ILS00, Rouymi [Rou11 and Ng Ng12. Peter Humphries has also shared a preprint with the author in which he independently obtains Theorem 3.1, and uses it to study low-lying zeros of the $L$-functions associated to $f \in$ $H_{\kappa}^{\star}(N, \epsilon)$. Theorem 3.1 is the only place in the proof where we have used the hypothesis $(N, m)=1$, in an essential way, and so is the source of the relatively prime conditions in Theorems 1.1 and 1.2 ,

We deal with (2) by appealing to the special value formula

$$
L\left(1, \mathrm{Ad}^{2} f\right)=\frac{\zeta^{(N)}(2)(4 \pi)^{\kappa}}{\Gamma(\kappa)} \frac{\|f\|_{L^{2}}^{2}}{\operatorname{Vol} X_{0}(N)},
$$

where $L\left(s, \operatorname{Ad}^{2} f\right)$ is a certain Dirichlet series whose coefficients involve $\lambda_{f}\left(n^{2}\right)$, and which we discuss in more detail in section 2. One may then swap the sum over $f$ and this Dirichlet series, and apply our Petersson formula for newforms (Theorem 3.1). Estimating the resulting sums directly using the Weil bound for $S_{\epsilon}(a, b, c)$ (see Lemma 4.2), one recovers that the trace of $T_{m}$ is $\ll_{m}(N \kappa)^{1+\varepsilon}$ (compare with (1.1)).

To save a bit more and obtain Theorem 1.1 we remove the weights $\|f\|_{L^{2}}^{2}$ more efficiently using a method due to Kowalski and Michel [KM99, Prop. 2]. Kowalski and Michel's method is based on Hölder's inequality and a large sieve inequality due to Duke and Kowalski [DK00, Thm. 4] for sub-families of automorphic forms on $\mathrm{GL}_{3}$. There are other notable large sieve inequalities for $\mathrm{GL}_{3}$ in the literature, see e.g. [BBM17, Thm. 3] and [Ven06, Thm. 1]. However, these two are not useful to us since we need a large sieve inequality which is efficient for the proper sub-family of $\mathrm{GL}_{3}$ forms cut out by the image of the adjoint square lift from $\mathrm{GL}_{2}$. The inequality of Duke and Kowalski is superior to the results [BBM17, Thm. 3] and [Ven06, Thm. 1] in the case of a thin subfamily and a long summation variable, which is the situation of interest to us.

1.4. Acknowledgements. I would like to thank Nathan Kaplan for a careful read and pointing out the Tsfasman-Vlăduţ-Zink theorem to me, Corentin Perret-Gentil for some helpful discussions, and the anonymous referee for a thorough and detailed report on the first version of this paper. 


\section{Preliminaries on $L$-Series}

If $L(s)$ is a meromorphic function defined in $\operatorname{Re}(s) \gg 1$ by an infinite product over primes $p$ of local factors $L_{p}(s)$, then for any integer $N$ we write

$$
L^{(N)}(s)=\prod_{p \nmid N} L_{p}(s)
$$

and

$$
L_{N}(s)=\prod_{p \mid N} L_{p}(s)
$$

so that $L(s)=L_{N}(s) L^{(N)}(s)$ for any $N \in \mathbf{N}$. To deal with the $\|f\|_{L^{2}}^{2}$-normalization alluded to in subsection 1.3, we introduce the "naive" adjoint square $L$-function. For $f \in H_{\kappa}^{\star}(N, \epsilon)$, let

$$
L\left(s, \operatorname{Ad}^{2} f\right)=\frac{\zeta^{(N)}(2 s)}{\zeta(s)} \sum_{n \geqslant 1} \frac{\left|\lambda_{f}(n)\right|^{2}}{n^{s}}=\prod_{p} L_{p}\left(s, \operatorname{Ad}^{2} f\right),
$$

where $\zeta(s)$ is the Riemann zeta function, and where

$$
L_{p}\left(s, \operatorname{Ad}^{2} f\right)= \begin{cases}\left(1-\frac{1}{p^{2 s}}\right)^{-1} \sum_{\alpha \geqslant 0} \frac{\bar{\epsilon}\left(p^{\alpha}\right) \lambda_{f}\left(p^{2 \alpha}\right)}{p^{\alpha s}} & \text { if } p \nmid N \\ \left(1-\frac{1}{p^{s}}\right)\left(1-\frac{\left|\lambda_{f}(p)\right|^{2}}{p^{s}}\right)^{-1} & \text { if } p \mid N .\end{cases}
$$

Warning: the $L\left(s, \mathrm{Ad}^{2} f\right)$ is not the true adjoint square $L$-function of $f$ as defined by functoriality (see [K04, pp. 133] and the online errata). But if $p \nmid N$, then $L_{p}\left(s, \operatorname{Ad}^{2} f\right)$ does match the local $L$ factor at $p$ of the true adjoint square $L$-function. Our "naive" adjoint square $L$ function $L\left(s, \operatorname{Ad}^{2} f\right)$ is chosen to be the Dirichlet series for which the following Lemma is true.

Lemma 2.1. The series $L\left(s, \mathrm{Ad}^{2} f\right)$ defined above is holomorphic for $\operatorname{Re}(s)>0$ and

$$
L\left(1, \operatorname{Ad}^{2} f\right)=\frac{\zeta^{(N)}(2)(4 \pi)^{\kappa}}{\Gamma(\kappa)} \frac{\langle f, f\rangle_{N}}{\operatorname{Vol} X_{0}(N)},
$$

where

and

$$
\langle f, f\rangle_{N}=\int_{\Gamma_{0}(N) \backslash \mathcal{H}}|f(z)|^{2} y^{\kappa} \frac{d x d y}{y^{2}}
$$

$$
\operatorname{Vol} X_{0}(N)=\int_{\Gamma_{0}(N) \backslash \mathcal{H}} \frac{d x d y}{y^{2}}=\frac{\pi}{3} \psi(N) .
$$

Proof. For the first statement, let $\pi$ denote the irreducible admissible cuspidal automorphic representation of $\mathrm{GL}_{2}$ generated by $f$, and denote by $L\left(s, \mathrm{Ad}^{2} \pi\right)$ the $L$-function of its adjoint square lift. We have by Gelbart and Jacquet [GJ78 that $L\left(s, \mathrm{Ad}^{2} \pi\right)$ is an entire function of $s$. Therefore, the prime-to- $N$ part of the naive $L$-function $L^{(N)}\left(s, \operatorname{Ad}^{2} f\right)$ is holomorphic for $\operatorname{Re}(s)>0$.

For the second statement, take the standard non-holomorphic Eisenstein series for $\Gamma_{0}(N)$ at the cusp $\infty$ given by

$$
E(z, s)=\sum_{\gamma \in \Gamma_{\infty} \backslash \Gamma_{0}(N)} \operatorname{Im}(\gamma z)^{s} .
$$

Then we have by the classical Rankin-Selberg unfolding argument

$$
\int_{\Gamma_{0}(N) \backslash \mathcal{H}}|f(z)|^{2} E(z, s) y^{\kappa} \frac{d x d y}{y^{2}}=\frac{\Gamma(s+\kappa-1)}{(4 \pi)^{s+\kappa-1}} \sum_{n \geqslant 1} \frac{\left|\lambda_{f}(n)\right|^{2}}{n^{s}} .
$$

We deduce the lemma by taking residues on both sides and recalling [Iwa97, Thm. 13.2] that

$$
\operatorname{Res}_{s=1} E(z, s)=\operatorname{Vol} X_{0}(N)^{-1} .
$$


Let $\varrho_{f}(n)$ be the Dirichlet series coefficients of $L^{(N)}\left(s, \operatorname{Ad}^{2} f\right)$. Explicitly,

$$
\varrho_{f}(n)= \begin{cases}\sum_{n=m^{2} \ell} \bar{\epsilon}(\ell) \lambda_{f}\left(\ell^{2}\right) & \text { if }(n, N)=1 \\ 0 & \text { if }(n, N)>1 .\end{cases}
$$

Inverting, we also have

$$
\bar{\epsilon}(n) \lambda_{f}\left(n^{2}\right)=\sum_{m^{2} \ell=n} \mu(m) \varrho_{f}(\ell) .
$$

For future reference, we write the partial sums of $L^{(N)}\left(1, \mathrm{Ad}^{2} f\right)$ compactly as

$$
\omega_{f}(x)=\sum_{n \leqslant x} \frac{\varrho_{f}(n)}{n} .
$$

By contrast, when $p \mid N$ we have that $L_{p}\left(s, \mathrm{Ad}^{2} f\right)$ is constant along $f \in H_{\kappa}^{\star}(N, \epsilon)$ by the following Lemma.

Lemma 2.2 (Ogg69 Thms 2,3). Let $p \mid N$ be a prime, and $\epsilon$ a Dirichlet character mod $N$. Write

$$
a_{N, \epsilon}(p)= \begin{cases}1 & \text { if } \epsilon \text { is not a character } \bmod N / p \\ \frac{1}{p} & \text { if } \epsilon \text { is a character } \bmod N / p \text { and } p^{2} \nmid N \\ 0 & \text { if } \epsilon \text { is a character } \bmod N / p \text { and } p^{2} \mid N .\end{cases}
$$

Then we have $\left|\lambda_{f}(p)\right|^{2}=a_{N, \epsilon}(p)$.

\section{Structural Steps}

We study the operator $T_{m}^{\prime}=T_{m} / m^{\frac{\kappa-1}{2}}$ on $S_{\kappa}\left(\Gamma_{0}(N), \epsilon\right)$ so that each eigenvalue $\lambda_{f}(m)$ of the $T_{m}^{\prime}$ operator is normalized by Deligne's theorem to have $\left|\lambda_{f}(m)\right| \leqslant d(m)$. We write $H_{\kappa}^{\star}(N, \epsilon)$ for the set of Hecke-normalized newforms in $S_{\kappa}(N, \epsilon)$ in the sense of Atkin-Lehner theory [AL70, Li75]. Also by Atkin-Lehner theory we have when $(m, N)=1$ that

$$
\operatorname{Tr}\left(T_{m}^{\prime} \mid S_{\kappa}\left(\Gamma_{0}(N), \epsilon\right)\right)=\sum_{L M=N} d(L) \sum_{f \in H_{\kappa}^{\star}(M, \epsilon)} \lambda_{f}(m)
$$

where we consider the interior sum to be empty if $\epsilon$ is not a character mod $M$. Thanks to (1.5), we can reduce the structural steps for traces on $S_{\kappa}(\Gamma(M, N))$ to the case of $S_{\kappa}\left(\Gamma_{0}(N), \epsilon\right)$.

Recall the notation from section 1.3 and write $c_{\kappa}=\Gamma(\kappa-1) /(4 \pi)^{\kappa-1}$. Let

$$
\Delta_{\kappa, N, \epsilon}(m, n)=\frac{c_{\kappa}}{(\sqrt{m n})^{\kappa-1}} \sum_{f \in \mathcal{B}_{\kappa}\left(\Gamma_{0}(N), \epsilon\right)} b_{f}(n) \overline{b_{f}(m)}
$$

so that the Petersson formula (1.10) is

$$
\Delta_{\kappa, N, \epsilon}(m, n)=\delta(m, n)+2 \pi i^{-\kappa} \sum_{\substack{c>0 \\ c \equiv 0(\bmod N)}} \frac{S_{\epsilon}(m, n, c)}{c} J_{\kappa-1}\left(\frac{4 \pi \sqrt{m n}}{c}\right) .
$$

The following theorem is our main tool for computing sums over the set of newforms $H_{\kappa}^{\star}(N, \epsilon)$.

Theorem 3.1. If $(m n, N)=1$ then we have

$$
c_{\kappa} \sum_{f \in H_{\kappa}^{\star}(N, \epsilon)} \frac{\overline{\lambda_{f}(m)} \lambda_{f}(n)}{\langle f, f\rangle_{N}}=\sum_{L M=N} \mu(L) R(M, L, \epsilon) \sum_{\substack{\ell \mid L^{\infty} \\(\ell, M)=1}} \frac{\bar{\epsilon}(\ell)}{\ell} \Delta_{\kappa, M, \epsilon}\left(m, n \ell^{2}\right),
$$


where

$$
R(M, L, \epsilon):=\frac{1}{L} \prod_{\substack{p^{2} \mid L \\ p \nmid M}}\left(1-\frac{1}{p^{2}}\right)^{-1} \prod_{p \mid(M, L)}\left(1-\frac{a_{M, \epsilon}(p)}{p}\right)^{-1},
$$

and $a_{M, \epsilon}(p)$ was defined in Lemma 2.2.

Proof. See section 7 .

Theorem 3.1 does not directly apply to (3.1) because of the normalization by $\langle f, f\rangle_{N}$.

We present a technique for removing the weights $\langle f, f\rangle_{N}$, which is a slight generalization of Kowalski and Michel [KM99, §3]. The idea for removing such weights first appeared in a paper of Murty Mur95. Let $\alpha=\left(\alpha_{f}\right)$ be a sequence of complex numbers indexed by

$$
f \in \bigcup_{N \geqslant 1} \bigcup_{\epsilon(\bmod N)} H_{\kappa}^{\star}(N, \epsilon) .
$$

Define the natural averaging operator

$$
A[\alpha]=A_{N, \epsilon}[\alpha]=\sum_{f \in H_{\kappa}^{\star}(N, \epsilon)} \alpha_{f}
$$

Let

$$
\omega_{f}=c_{\kappa} \frac{L_{N}\left(1, \mathrm{Ad}^{2} f\right)}{\langle f, f\rangle_{N}} .
$$

Then we define the harmonic averaging operator

$$
A^{h}[\alpha]=A_{N, \epsilon}^{h}[\alpha]=\sum_{f \in H_{\kappa}^{\star}(N, \epsilon)} \omega_{f} \alpha_{f} .
$$

The following proposition is a minor generalization of Proposition 2 of [KM99. It allows us to pass from natural averages of newforms to harmonic averages of newforms.

Proposition 3.2. Let $\alpha=\left(\alpha_{f}\right)$ be a sequence of complex numbers indexed by $f \in H_{\kappa}^{\star}(N, \epsilon)$ running over all $N$ and all $\epsilon$. Suppose that for all $\varepsilon>0$

$$
A^{h}\left[\left|\alpha_{f}\right|\right] \ll_{\varepsilon}(N \kappa)^{\varepsilon}
$$

and

$$
\max _{f \in H_{\kappa}^{\star}(N, \epsilon)}\left|\omega_{f} \alpha_{f}\right| \ll(N \kappa)^{-\delta+\varepsilon}
$$

for some absolute $\delta>0$. For any integer $r \geqslant 1$ write $x=(N \kappa)^{\frac{10}{r}}$. Then we have

$$
A\left[\alpha_{f}\right]=\frac{\kappa-1}{4 \pi} \frac{\operatorname{Vol} X_{0}(N)}{\zeta^{(N)}(2)}\left(A^{h}\left[\omega_{f}(x) \alpha_{f}\right]+O_{\varepsilon, r}\left(x^{-\frac{\delta}{20}+\varepsilon}+(N \kappa)^{-1}\right)\right) .
$$

Proof. See section 6.

One of the main ingredients in the proof of Proposition 3.2 is a large sieve inequality for the Dirichlet series coefficients of the automorphic adjoint square $L$-funciton $L\left(s, \mathrm{Ad}^{2} \pi\right)$, see Proposition [6.2, which is a quotation of [DK00, Cor 6]. This inequality is only valid when the length of summation $X$ satisfies $X \gg(N \kappa)^{8}$, which is far from the expected truth. Nonetheless, as of now it is the best available such inequality in the range of parameters of interest to us. The exponent $-\delta / 20$ in Proposition 3.2 is optimized given the exponent 8 above, and any improvement over the result of Duke and Kowalski would lead to a corresponding improvement to the value $20=2(8+2)$. 
We apply Proposition 3.2 with $\alpha_{f}=\overline{\lambda_{f}}(m)$ to equation (3.1) to get

$$
\begin{array}{r}
\overline{\operatorname{Tr}\left(T_{m}^{\prime} \mid S_{\kappa}\left(\Gamma_{0}(N), \epsilon\right)\right)}=\sum_{L M=N} d(L) \frac{\kappa-1}{4 \pi} \frac{\operatorname{Vol} X_{0}(M)}{\zeta^{(M)}(2)} A_{M, \epsilon}^{h}\left[\omega_{f}(x) \overline{\lambda_{f}(m)}\right]+O\left(\kappa N^{1+\varepsilon} x^{-\delta / 20+\varepsilon}+N^{\varepsilon}\right) \\
=\frac{\kappa-1}{12} \sum_{L M=N} \frac{d(L) \psi(M)}{\zeta^{(M)}(2)} \sum_{\substack{n \leqslant x \\
(n, M)=1}} \frac{1}{n} \sum_{n=k^{2} \ell} \bar{\epsilon}(\ell) A_{M, \epsilon}^{h}\left[\overline{\lambda_{f}(m)} \lambda_{f}\left(\ell^{2}\right)\right] \\
+O\left(\kappa N^{1+\varepsilon} x^{-\delta / 20+\varepsilon}+N^{\varepsilon}\right) .
\end{array}
$$

We are now ready to apply Theorem 3.1. We deduce a version of the newform formula for the harmonic averages $A^{h}\left[\overline{\lambda_{f}(m)} \lambda_{f}(n)\right]$ appearing in (3.5).

Lemma 3.3. Let $\mathbf{c}_{p}(\epsilon)$ denote the exponent of the p-part of $\mathbf{c}(\epsilon)$. If $(m n, N)=1$ then we have

$$
A_{N, \epsilon}^{h}\left[\overline{\lambda_{f}(m)} \lambda_{f}(n)\right]=\frac{1}{\psi(N)} \sum_{L M=N} \mu(L) M F(M, \epsilon) \prod_{p^{2} \mid M}\left(1-\frac{1}{p^{2}}\right) \sum_{\substack{\ell \mid L^{\infty} \\(\ell, M)=1}} \frac{\bar{\epsilon}(\ell)}{\ell} \Delta_{\kappa, M, \epsilon}\left(m, n \ell^{2}\right),
$$

where

$$
F(M, \epsilon)=\prod_{\substack{p \| M \\ \mathbf{c}_{p}(\epsilon)=1}}\left(1+\frac{1}{p}\right) \prod_{\substack{p^{\alpha} \| M \\ \alpha \geqslant 2 \\ \mathbf{c}_{p}(\epsilon)=\alpha}}\left(1-\frac{1}{p}\right)^{-1} .
$$

In particular, if $\epsilon=\epsilon_{0}$ is trivial we have

$$
A_{N, \epsilon_{0}}^{h}\left[\overline{\lambda_{f}(m)} \lambda_{f}(n)\right]=\frac{1}{\psi(N)} \sum_{L M=N} \mu(L) M \prod_{p^{2} \mid M}\left(1-\frac{1}{p^{2}}\right) \sum_{\substack{\ell \mid L^{\infty} \\(\ell, M)=1}} \frac{1}{\ell} \Delta_{\kappa, M, \epsilon_{0}}\left(m, n \ell^{2}\right) .
$$

Note that formula (3.7) resembles closely the formula found in $\mathrm{BBD}^{+} 17$, Prop. 4.1].

Proof. By the definition of $L_{p}\left(1, \mathrm{Ad}^{2} f\right)$ and Theorem 3.1 we have

$$
A^{h}\left[\overline{\lambda_{f}(m)} \lambda_{f}(n)\right]=\prod_{p \mid N}\left(1-\frac{1}{p}\right)\left(1-\frac{a_{N, \epsilon}(p)}{p}\right)^{-1} \sum_{L M=N} \mu(L) R(M, L, \epsilon) \sum_{\substack{\ell \mid L^{\infty} \\(\ell, M)=1}} \frac{\bar{\epsilon}(\ell)}{\ell} \Delta_{\kappa, M, \epsilon}\left(m, n \ell^{2}\right) .
$$

It suffices to show for any $L, M$ that

$$
\frac{\psi(L M)}{M} \prod_{p \mid L M}\left(1-\frac{1}{p}\right)\left(1-\frac{a_{L M, \epsilon}(p)}{p}\right)^{-1} R(M, L, \epsilon)=\prod_{p^{2} \mid M}\left(1-\frac{1}{p^{2}}\right) F(M, \epsilon) .
$$

We may also assume that $c(\epsilon) \mid M$, since otherwise $\Delta_{\kappa, M, \epsilon}\left(m, n \ell^{2}\right)=0$. Both sides of (3.8) are multiplicative, so it suffices to check the case $M=p^{\alpha}$ and $L=p^{\beta}$ for an arbitrary prime $p$. The following cases can be easily verified one-by-one.

- $\alpha \geqslant 2, \beta \geqslant 1$, and $\mathbf{c}_{p}(\epsilon)=\alpha$

- $\alpha \geqslant 2, \beta \geqslant 1$, and $\mathbf{c}_{p}(\epsilon)<\alpha$

- $\alpha \geqslant 2, \beta=0$, and $\mathbf{c}_{p}(\epsilon)=\alpha$

- $\alpha \geqslant 2, \beta=0$, and $\mathbf{c}_{p}(\epsilon)<\alpha$

- $\alpha=1, \beta \geqslant 1$, and $\mathbf{c}_{p}(\epsilon)=1$

- $\alpha=1, \beta \geqslant 1$, and $\mathbf{c}_{p}(\epsilon)=0$

- $\alpha=1, \beta=0$, and $\mathbf{c}_{p}(\epsilon)=1$ 
- $\alpha=1, \beta=0$, and $\mathbf{c}_{p}(\epsilon)=0$

- $\alpha=0, \beta \geqslant 2$, and $\mathbf{c}_{p}(\epsilon)=0$

- $\alpha=0, \beta=1$, and $\mathbf{c}_{p}(\epsilon)=0$.

\section{Analysis FOR $\Gamma_{0}(N)$}

Now we put together (3.5), the newform formula (3.6), and the Petersson formula (3.2). By (3.5) and (3.6) we have that

$$
\overline{\operatorname{Tr}\left(T_{m}^{\prime} \mid S_{\kappa}\left(\Gamma_{0}(N), \epsilon\right)\right)}=A+E,
$$

where for an integer $r \geqslant 1$ to be chosen later we set $x^{r}=(N \kappa)^{10}$ and have

$$
\begin{array}{r}
A=\frac{\kappa-1}{12} \sum_{L M=N} \frac{d(L)}{\zeta^{(M)}(2)} \sum_{\substack{k \leqslant x^{1 / 2} \\
(k, M)=1}} \frac{1}{k^{2}} \sum_{\substack{\ell \leqslant x / k^{2} \\
(\ell, M)=1}} \frac{\bar{\epsilon}(\ell)}{\ell} \sum_{W Q=M} \mu(Q) W F(W, \epsilon) \prod_{p^{2} \mid W}\left(1-\frac{1}{p^{2}}\right) \\
\times \sum_{\substack{q \mid Q^{\infty} \\
(q, W)=1}} \frac{\bar{\epsilon}(q)}{q} \Delta_{\kappa, W, \epsilon}\left(m, q^{2} \ell^{2}\right),
\end{array}
$$

and $E$ is the error term from (3.5) of size

$$
E \ll_{r, \varepsilon} \kappa N^{1+\varepsilon} x^{-\frac{\delta}{20}+\varepsilon}+N^{\varepsilon} .
$$

Applying (3.2) to $A$ we get that

$$
A=D+O D
$$

where $D$ and $O D$ are the contributions from the diagonal term and off-diagonal term of (3.2), respectively. We insert $\delta_{m=q^{2} \ell^{2}} \delta_{\mathbf{c}(\epsilon) \mid W}$ for $\Delta_{\kappa, W, \epsilon}\left(m, q^{2} \ell^{2}\right)$ in (4.1) to find

$$
D=\frac{\kappa-1}{12} \frac{\bar{\epsilon}\left(m^{\frac{1}{2}}\right)}{m^{\frac{1}{2}}} \sum_{L M=N} \frac{d(L)}{\zeta^{(M)}(2)} \sum_{\substack{k \leqslant x^{1 / 2} / m^{1 / 4} \\(k, M)=1}} \frac{1}{k^{2}} \sum_{W Q=M} \mu(Q) W F(W, \epsilon) \prod_{p^{2} \mid W}\left(1-\frac{1}{p^{2}}\right) \delta_{\mathbf{c}(\epsilon) \mid W} .
$$

Extending the sum over $k$ to infinity we conclude that

$$
D=\frac{\kappa-1}{12} \frac{\bar{\epsilon}\left(m^{\frac{1}{2}}\right)}{m^{\frac{1}{2}}} \sum_{L M=N} d(L) \sum_{W Q=M} \mu(Q) W F(W, \epsilon) \prod_{p^{2} \mid W}\left(1-\frac{1}{p^{2}}\right) \delta_{\mathbf{c}(\epsilon) \mid W}+O_{\varepsilon}\left(\frac{\kappa N^{1+\varepsilon}}{x^{\frac{1}{2}} m^{\frac{1}{4}}}\left|\epsilon\left(m^{\frac{1}{2}}\right)\right|\right) .
$$

By a tedious case check on prime powers we have

$$
\psi(N) \delta_{\mathbf{c}(\epsilon) \mid N}=\sum_{L M=N} M F(M, \epsilon) \delta_{\mathbf{c}(\epsilon) \mid M} \prod_{p^{2} \mid M}\left(1-\frac{1}{p^{2}}\right) .
$$

Therefore the result of the diagonal contribution is

$$
D=\frac{\kappa-1}{12} \frac{\bar{\epsilon}\left(m^{\frac{1}{2}}\right)}{m^{\frac{1}{2}}} \psi(N)+O\left(\frac{\kappa N^{1+\varepsilon}}{x^{\frac{1}{2}} m^{\frac{1}{4}}}\left|\epsilon\left(m^{\frac{1}{2}}\right)\right|\right),
$$

which matches what one finds directly from the identity contribution of the Eichler-Selberg trace formula.

Now we treat the off-diagonal terms. Let

$$
B(Y, m, W)=\sum_{\substack{\ell \leqslant Y \\(\ell, M)=1}} \sum_{\substack{q \mid Q^{\infty} \\(q, W)=1}} \frac{\bar{\epsilon}(q \ell)}{q \ell} \sum_{c \equiv 0(\bmod W)} \frac{S_{\epsilon}\left(m, q^{2} \ell^{2}, c\right)}{c} J_{\kappa-1}\left(\frac{4 \pi q \ell \sqrt{m}}{c}\right) .
$$


Then we have that

$$
O D=\frac{\kappa-1}{12} \sum_{L M=N} \frac{d(L)}{\zeta^{(M)}(2)} \sum_{W Q=M} \mu(Q) W F(W, \epsilon) \prod_{p^{2} \mid W}\left(1-\frac{1}{p^{2}}\right) \sum_{\substack{k \leqslant x^{1 / 2} \\(k, M)=1}} \frac{1}{k^{2}} B\left(x / k^{2}, m, W\right) .
$$

Lemma 4.1. Let $d_{3}$ denote the 3 -divisor function. For any $m, n \geqslant 1$ we have

$$
\begin{aligned}
& \sum_{c \equiv 0(\bmod W)} \frac{S_{\epsilon}(m, n, c)}{c} J_{\kappa-1}\left(\frac{4 \pi \sqrt{m n}}{c}\right) \\
& \ll \mathbf{c}(\epsilon)^{\frac{1}{4}} \prod_{p \mid \mathbf{c}(\epsilon)} p^{\frac{1}{4}} \frac{(m, n, W)^{\frac{1}{2}} d_{3}((m, n)) d(W)}{W \kappa^{\frac{5}{6}}}\left(\frac{m n}{\sqrt{m n}+\kappa W}\right)^{\frac{1}{2}} \log 2 m n .
\end{aligned}
$$

Proof. The proof is identical to [LS00, Cor. 2.2] but with the following bound on the Kloosterman sum in lieu of the standard bound without nebentype character.

Lemma 4.2. For integers $c \in N \mathbf{Z}$ and $a, b \in \mathbf{Z}$ with $c \neq 0$ and $\mathbf{c}(\epsilon) \mid N$, we have the estimate

$$
\left|S_{\epsilon}(a, b ; c)\right| \leqslant d(c)(a, b, c)^{\frac{1}{2}} c^{\frac{1}{2}} \mathbf{c}(\epsilon)^{\frac{1}{4}} \mathbf{c}^{*}(\epsilon)^{\frac{1}{4}} .
$$

Proof. See Knightly and Li [KL13, Thm. 9.2].

Applying Lemma 4.1 and estimating sums by integrals we find

$$
B(Y, m, W) \ll \mathbf{c}(\epsilon)^{\frac{1}{4}} \mathbf{c}^{*}(\epsilon)^{\frac{1}{4}} \frac{d(W) m^{\frac{1}{4}} Y^{\frac{1}{2}}}{W \kappa^{\frac{5}{6}}} \log m Y,
$$

hence one estimates that

$$
O D \ll_{\varepsilon} \mathbf{c}(\epsilon)^{\frac{1}{4}} \mathbf{c}^{*}(\epsilon)^{\frac{1}{4}} x^{\frac{1}{2}} \kappa^{\frac{1}{6}} m^{\frac{1}{4}} N^{\varepsilon} \log m x .
$$

We have $\operatorname{Tr}\left(T_{m}^{\prime} \mid S_{\kappa}\left(\Gamma_{0}(N, \epsilon)\right)\right)=\bar{D}+\overline{O D}+\bar{E}$, and so collecting error terms we obtain

$$
\begin{aligned}
\operatorname{Tr}\left(T_{m}^{\prime} \mid S_{\kappa}\left(\Gamma_{0}(N, \epsilon)\right)\right)=\frac{\kappa-1}{12} \frac{\epsilon\left(m^{\frac{1}{2}}\right)}{m^{\frac{1}{2}}} \psi(N)+O_{\varepsilon}\left(\mathbf{c}(\epsilon)^{\frac{1}{4}} \mathbf{c}^{*}(\epsilon)^{\frac{1}{4}} x^{\frac{1}{2}} \kappa^{\frac{1}{6}} m^{\frac{1}{4}} N^{\varepsilon} \log m x\right. & \\
& \left.+\kappa N^{1+\varepsilon} x^{-\frac{\delta}{20}+\varepsilon}+N^{\varepsilon}\right) .
\end{aligned}
$$

We now optimize the value of $r$. By [GHL94, Ban97, the exponent $\delta=1$ is admissible. The error in (4.5) is minimized when

$$
x^{\frac{11}{20}}=\frac{N \kappa^{\frac{5}{6}}}{m^{\frac{1}{4}} \mathbf{c}(\epsilon)^{\frac{1}{4}} \mathbf{c}^{*}(\epsilon)^{\frac{1}{4}}} .
$$

Let us assume that there is some $\eta>0$ such that

$$
m^{\frac{1}{4}} \mathbf{c}(\epsilon)^{\frac{1}{4}} \mathbf{c}^{*}(\epsilon)^{\frac{1}{4}} \ll\left(N \kappa^{\frac{5}{6}}\right)^{1-\eta} .
$$

We choose $r \geqslant 1$ to be the nearest integer to

$$
\frac{11}{2}\left(1+\frac{\log \left(\kappa^{\frac{1}{6}} m^{\frac{1}{4}} \mathbf{c}(\epsilon)^{\frac{1}{4}} \mathbf{c}^{*}(\epsilon)^{\frac{1}{4}}\right)}{\log \left(N \kappa^{\frac{5}{6}}\right)-\log \left(m^{\frac{1}{4}} \mathbf{c}(\epsilon)^{\frac{1}{4}} \mathbf{c}^{*}(\epsilon)^{\frac{1}{4}}\right)}\right),
$$

which by (4.6) is then bounded above uniformly in terms of $\eta>0$ only. 


\section{Analysis for $\Gamma(M, N)$}

Recall from (1.5) that

$$
\operatorname{Tr}\left(\langle\bar{d}\rangle T_{m}^{\prime} \mid S_{\kappa}(\Gamma(M, N))\right)=\sum_{\epsilon(\bmod N)} \bar{\epsilon}(d) \operatorname{Tr}\left(T_{m}^{\prime} \mid S_{\kappa}\left(\Gamma_{0}(M N), \epsilon\right)\right),
$$

and that in section 4 we decomposed the interior of this as

$$
\overline{\operatorname{Tr}\left(T_{m}^{\prime} \mid S_{\kappa}\left(\Gamma_{0}(M N), \epsilon\right)\right)}=D+O D+E .
$$

Summing the formula (4.3) for $D$ and (4.2) for $E$ trivially over characters $\epsilon(\bmod N)$ we get

$$
\begin{array}{r}
\operatorname{Tr}\left(\langle\bar{d}\rangle T_{m}^{\prime} \mid S_{\kappa}(\Gamma(M, N))\right)=\frac{\kappa-1}{24} m^{-\frac{1}{2}} \varphi(N) \psi(N M)\left(\delta_{N}\left(m^{\frac{1}{2}} d, 1\right)+(-1)^{\kappa} \delta_{N}\left(m^{\frac{1}{2}} d,-1\right)\right) \\
+\overline{O D^{*}}+O_{\eta, \varepsilon}\left(\kappa\left(M N^{2}\right)^{1+\varepsilon} x^{-\frac{\delta}{20}+\varepsilon}+N(M N)^{\varepsilon}\right),
\end{array}
$$

where $x^{r}=(M N \kappa)^{10}, r$ is a parameter to be chosen later, and

$$
O D^{*}=\sum_{\substack{\epsilon(\bmod N) \\ \epsilon(-1)=(-1)^{\kappa}}} \epsilon(d) O D
$$

Let

$$
\begin{aligned}
B^{*}(Y, m, W)=\sum_{\substack{\epsilon(\bmod N) \\
\epsilon(-1)=(-1)^{\kappa}}} \epsilon(d) F(W, \epsilon) \sum_{\substack{(\ell, K)=1 \\
\ell \leqslant Y}} \sum_{\substack{q \mid Q^{\infty} \\
(q, W)=1}} \frac{\overline{\epsilon(q \ell)}}{q \ell} \sum_{c \equiv 0(\bmod W)} \frac{S_{\epsilon}\left(m, q^{2} \ell^{2}, c\right)}{c} \\
\times J_{\kappa-1}\left(\frac{4 \pi \ell q \sqrt{m}}{c}\right),
\end{aligned}
$$

so that we have

$$
O D^{*}=\frac{\kappa-1}{12} \sum_{L K=M N} \frac{d(L)}{\zeta^{(K)}(2)} \sum_{\substack{\left.k \leqslant x^{1 / 2} \\ k, K\right)=1}} \frac{1}{k^{2}} \sum_{W Q=K} \mu(Q) W \prod_{p^{2} \mid W}\left(1-\frac{1}{p^{2}}\right) B^{*}\left(x / k^{2}, m, W\right) .
$$

We would like to utilize the orthogonality of characters over $\epsilon(\bmod N)$. To implement this, we now refresh the notation. Suppose $W, N \geqslant 1$ are integers such that $W \mid N^{2}$. For $a, b, d, \kappa \in \mathbf{Z}$ and $1 \leqslant c \equiv 0(\bmod W)$ define

$$
T_{W}(a, b, c):=\sum_{\substack{\epsilon(\bmod N) \\ \epsilon(-1)=(-1)^{\kappa} \\ \mathbf{c}(\epsilon) \mid W}} \epsilon(d) \bar{\epsilon}(b) F(W, \epsilon) S_{\epsilon}(a, b, c) .
$$

With this notation, we have

$$
B^{*}(Y, m, W)=\sum_{\substack{(\ell, K)=1 \\ \ell \leqslant Y}} \sum_{\substack{q \mid Q^{\infty} \\(q, W)=1}} \frac{1}{q \ell} \sum_{c \equiv 0(\bmod W)} \frac{T_{W}\left(m, q^{2} \ell^{2}, c\right)}{c} J_{\kappa-1}\left(\frac{4 \pi \ell q \sqrt{m}}{c}\right) .
$$

We can derive a bound on $T_{W}$ by appealing to the Weil bound for Kloosterman sums.

Lemma 5.1. Suppose $W, N \geqslant 1$ such that $W \mid N^{2}, a, b, d, \kappa \in \mathbf{Z}$ such that $(b, W)=1,(d, N)=1$, and $1 \leqslant c \equiv 0(\bmod W)$. We factor $c=c_{1} c_{2}$ with $c_{1} \mid W^{\infty}$ and $\left(c_{2}, W\right)=1$. Then

$$
\left|T_{W}(a, b, c)\right| \leqslant \psi\left(c_{1}\right) d\left(c_{2}\right)\left(a, b, c_{2}\right)^{1 / 2} c_{2}^{1 / 2} .
$$


Proof. Consider the sum

$$
T_{W}^{\prime}(a, b, c):=\sum_{\substack{\epsilon(\bmod N) \\ \mathbf{c}(\epsilon) \mid W}} \epsilon(d) \bar{\epsilon}(b) F(W, \epsilon) S_{\epsilon}(a, b, c),
$$

which is a minor variation of $T_{W}(a, b, c)$, but omitting the global condition $\epsilon(-1)=(-1)^{\kappa}$. We first consider the sum $T_{W}^{\prime}$ locally, returning to $T_{W}$ at the end of the proof. Let $\alpha, \beta, \gamma \geqslant 0$ such that $\alpha \leqslant \gamma, \alpha \leqslant 2 \beta,\left(d, p^{\beta}\right)=\left(b, p^{\alpha}\right)=1$, and consider $T_{p^{\alpha}}^{\prime}\left(a, b, p^{\gamma}\right)$. Let

$$
I(\alpha, \beta):=\sum_{\epsilon\left(\bmod p^{\beta}\right)} \epsilon(d x) \bar{\epsilon}(b) \delta_{\mathbf{c}_{p}(\epsilon) \leqslant \alpha} \begin{cases}1+\frac{1}{p} & \text { if } \mathbf{c}_{p}(\epsilon)=\alpha=1 \\ \left(1-\frac{1}{p}\right)^{-1} & \text { if } \mathbf{c}_{p}(\epsilon)=\alpha \geqslant 2 \\ 1 & \text { else. }\end{cases}
$$

By opening the Kloosterman sum and exchanging order of summation we have

$$
T_{p^{\alpha}}^{\prime}\left(a, b, p^{\gamma}\right)=\sum_{x\left(\bmod p^{\gamma}\right)}^{*} e\left(\frac{a x+b \bar{x}}{p^{\gamma}}\right) I(\alpha, \beta) .
$$

Next we break into four cases:

(1) $\alpha>\beta$

(2) $0=\alpha \leqslant \beta$

(3) $1=\alpha \leqslant \beta$

(4) $2 \leqslant \alpha \leqslant \beta$.

Recall the orthogonality relation

$$
\sum_{\epsilon(\bmod n)} \epsilon(a) \bar{\epsilon}(b)=\varphi(n) \delta_{n}(a, b)
$$

and the almost-orthogonality relation (see e.g. [HB81, Section 2])

$$
\sum_{\mathbf{c}(\epsilon)=c} \epsilon(a) \bar{\epsilon}(b)=\sum_{\delta \mid(a-b, c)} \varphi(\delta) \mu\left(\frac{c}{\delta}\right) .
$$

We apply these to evaluate $I(\alpha, \beta)$ in cases (11)-(4). We find

$$
I(\alpha, \beta)= \begin{cases}\varphi\left(p^{\beta}\right) \delta_{p^{\beta}}(x d, b) & \text { if } \alpha>\beta \\ 1 & \text { if } 0=\alpha \leqslant \beta \\ \varphi(p) \delta_{p}(x d, b)+\frac{1}{p} \sum_{\delta \mid(p, x d-b)} \varphi(\delta) \mu\left(p^{\gamma} / \delta\right) & \text { if } 1=\alpha \leqslant \beta \\ \varphi\left(p^{\alpha}\right) \delta_{p^{\alpha}}(x d, b)+\frac{1}{p-1} \sum_{\delta \mid\left(p^{\alpha}, x d-b\right)} \varphi(\delta) \mu\left(p^{\gamma} / \delta\right) & \text { if } 1=\alpha \leqslant \beta .\end{cases}
$$

Recall that $\left(d, p^{\beta}\right)=1$, so that $d^{-1}\left(\bmod p^{\beta}\right)\left(\operatorname{or}\left(\bmod p^{\gamma}\right)\right.$ in cases (3) and (4) $)$ exists. Inserting (5.5) to (5.4), we find the following.

Case (11): $\beta<\alpha$.

$$
T_{p^{\alpha}}^{\prime}\left(a, b, p^{\gamma}\right)=\varphi\left(p^{\beta}\right) \sum_{\substack{x\left(\bmod p^{\gamma}\right) \\ x \equiv d^{-1} b\left(\bmod p^{\beta}\right)}}^{*} e\left(\frac{a x+b \bar{x}}{p^{\gamma}}\right) .
$$

Case (21): $\beta \geqslant \alpha=0$.

$$
T_{1}^{\prime}\left(a, b, p^{\gamma}\right)=\sum_{x\left(\bmod p^{\gamma}\right)}^{*} e\left(\frac{a x+b \bar{x}}{p^{\gamma}}\right)=S\left(a, b, p^{\gamma}\right) .
$$


Case (3): $\beta \geqslant \alpha=1$.

$$
T_{p}^{\prime}\left(a, b, p^{\gamma}\right)=\varphi(p) \sum_{\substack{x\left(\bmod p^{\gamma}\right) \\ x \equiv d^{-1} b(\bmod p)}}^{*} e\left(\frac{a x+b \bar{x}}{p^{\gamma}}\right)+\frac{1}{p} \sum_{\delta \mid p} \varphi(\delta) \mu(p / \delta) \sum_{\substack{x\left(\bmod p^{\gamma}\right) \\ x \equiv d^{-1} b(\bmod \delta)}}^{*} e\left(\frac{a x+b \bar{x}}{p^{\gamma}}\right) .
$$

Case (41): $\beta \geqslant \alpha \geqslant 2$.

$$
T_{p^{\alpha}}^{\prime}\left(a, b, p^{\gamma}\right)=\varphi\left(p^{\alpha}\right) \sum_{\substack{x\left(\bmod p^{\gamma}\right) \\ x \equiv d^{-1} b\left(\bmod p^{\alpha}\right)}}^{*} e\left(\frac{a x+b \bar{x}}{p^{\gamma}}\right)+\frac{1}{p-1} \sum_{\delta \mid p^{\alpha}} \varphi(\delta) \mu\left(p^{\alpha} / \delta\right) \sum_{\substack{x\left(\bmod p^{\gamma}\right) \\ x \equiv d^{-1} b(\bmod \delta)}}^{*} e\left(\frac{a x+b \bar{x}}{p^{\gamma}}\right) .
$$

Using the Weil bound for Kloosterman sums and trivial bounds, we find for all integers $a, b$, and non-zero integers $0 \leqslant i \leqslant j$, and $(y, p)=1$ we have

$$
\left|\sum_{\substack{x\left(\bmod p^{j}\right) \\ x \equiv y\left(\bmod p^{i}\right)}}^{*} e\left(\frac{a x+b^{2} \bar{x}}{p^{\gamma}}\right)\right| \leqslant \begin{cases}d\left(p^{j}\right)\left(a, b^{2}, p^{j}\right)^{\frac{1}{2}} \sqrt{p^{j}} & \text { if } i=0 \\ p^{j-i} & \text { else. }\end{cases}
$$

Applying (5.6) to the various cases above, we find that cases (11), (3), and (4), i.e. when $\alpha>0$, the bound

$$
\left|T_{p^{\alpha}}^{\prime}\left(a, b, p^{\gamma}\right)\right| \leqslant \psi\left(p^{\gamma}\right)
$$

In case (2), i.e. when $\alpha=0$, we have

$$
\left|T_{p^{\alpha}}^{\prime}\left(a, b, p^{\gamma}\right)\right| \leqslant d\left(p^{\gamma}\right)\left(a, b, p^{\gamma}\right)^{1 / 2} p^{\gamma / 2} .
$$

Thus the estimation of $T_{p^{\alpha}}^{\prime}\left(a, b, p^{\gamma}\right)$ is finished.

Now we return to the case of $T_{W}(a, b, c)$. We have

$$
T_{W}(a, b, c)=\frac{1}{2} T_{W}^{\prime}(a, b, c)+\frac{(-1)^{\kappa}}{2} T_{W}^{\prime}(a,-b, c),
$$

so it suffices to establish the bound stated in the lemma for $T_{W}^{\prime}(a, b, c)$. We have that $T_{W}^{\prime}(a, b, c)$ is twisted multiplicative, i.e. we have a factorization

$$
T_{W}^{\prime}(a, b, c)=\prod_{\substack{p^{\alpha}\left\|W \\ p^{\gamma}\right\| c}} T_{p^{\alpha}}^{\prime}\left(a \overline{c p^{-\gamma}}, b \overline{c p^{-\gamma}}, p^{\gamma}\right) .
$$

Bounding the left hand side of (5.9) using (5.7) and (5.8), we conclude the proof of the lemma.

Applying Lemma 5.1 to (5.3) we get

$$
B^{*}(Y, m, W) \leqslant \sum_{\substack{(\ell, K)=1 \\ \ell \leqslant Y}} \sum_{\substack{q \mid Q^{\infty} \\(q, W)=1}} \frac{1}{q \ell} \sum_{W\left|c_{1}\right| W^{\infty}} \frac{\psi\left(c_{1}\right)}{c_{1}} \sum_{\left(c_{2}, W\right)=1} \frac{d\left(c_{2}\right)\left(m, q^{2} \ell^{2}, c_{2}\right)^{\frac{1}{2}}}{\sqrt{c_{2}}}\left|J_{\kappa-1}\left(\frac{4 \pi q \ell \sqrt{m}}{c_{1} c_{2}}\right)\right| .
$$

Again following closely the proof of [ILS00, Cor. 2.2] we have that

$$
\sum_{\left(c_{2}, W\right)=1} \frac{d\left(c_{2}\right)\left(a, b^{2}, c_{2}\right)^{\frac{1}{2}}}{\sqrt{c_{2}}}\left|J_{\kappa-1}\left(\frac{4 \pi b \sqrt{a}}{c_{1} c_{2}}\right)\right| \ll \frac{d_{3}\left(\left(a, b^{2}\right)\right)}{\kappa^{\frac{5}{6}} \sqrt{c_{1}}}\left(\frac{b^{2} a}{b \sqrt{a}+c_{1} \kappa}\right)^{\frac{1}{2}} \log 2 b^{2} a .
$$

We have moreover that

$$
\sum_{W\left|c_{1}\right| W^{\infty}} \frac{1}{\sqrt{c_{1}}} \frac{1}{\left(b \sqrt{a}+c_{1} \kappa\right)^{\frac{1}{2}}} \leqslant \frac{2}{W^{\frac{1}{2}} b^{\frac{1}{2}} a^{\frac{1}{4}}} .
$$


These last two estimations lead to

$$
\begin{aligned}
B^{*}(Y, m, W) & \ll \frac{m^{\frac{1}{4}}}{\kappa^{\frac{5}{6}}} \frac{\psi(W)}{W^{\frac{3}{2}}} \sum_{\substack{(\ell, K)=1 \\
\ell \leqslant Y}} \sum_{\substack{q \mid Q^{\infty} \\
(q, W)=1}} \frac{d_{3}\left(\left(m, q^{2} \ell^{2}\right)\right)}{\sqrt{q \ell}} \log \left(2 q^{2} \ell^{2} m\right) \\
& \ll \frac{m^{\frac{1}{4}} Y^{\frac{1}{2}}(\log Y)^{3} \log 2 m}{\kappa^{\frac{5}{6}}} \frac{\psi(W)}{W^{\frac{3}{2}}} .
\end{aligned}
$$

Inserting this into (5.2) we get

$$
O D^{*} \ll \kappa^{\frac{1}{6}} M N^{\frac{1}{2}+\varepsilon} x^{\frac{1}{2}}(\log x)^{3} m^{\frac{1}{4}} \log 2 m,
$$

and inserting this into (5.1) we conclude that

$$
\begin{array}{r}
\operatorname{Tr}\left(\langle\bar{d}\rangle T_{m}^{\prime} \mid S_{\kappa}(\Gamma(M, N))\right)=\frac{\kappa-1}{24} m^{-\frac{1}{2}} \varphi(N) \psi(N M)\left(\delta_{N}\left(m^{\frac{1}{2}} d, 1\right)+(-1)^{\kappa} \delta_{N}\left(m^{\frac{1}{2}} d,-1\right)\right) \\
+O_{\eta, \varepsilon}\left(\kappa^{\frac{1}{6} M N^{\frac{1}{2}}} x^{\frac{1}{2}+\varepsilon} m^{\frac{1}{4}} \log 2 m+\kappa\left(M N^{2}\right)^{1+\varepsilon} x^{-\frac{\delta}{20}+\varepsilon}+N(M N)^{\varepsilon}\right) .
\end{array}
$$

Now we optimize the value of $r$. The error term is minimized when

$$
x^{\frac{11}{20}}=\frac{N^{\frac{3}{2}} \kappa^{\frac{5}{6}}}{m^{\frac{1}{4}}} .
$$

Let us assume that there is some $\eta>0$ such that

$$
m^{\frac{1}{4}} \ll\left(N^{\frac{3}{2}} \kappa^{\frac{5}{6}}\right)^{1-\eta} .
$$

We choose $r \geqslant 1$ to be the nearest integer to

$$
\frac{11}{2}\left(\frac{\log M N \kappa}{\log N^{\frac{3}{2}} \kappa^{\frac{5}{6}}-\log m^{\frac{1}{4}}}\right)
$$

which is then bounded above uniformly in terms of $\eta>0$ only.

\section{Proof of Proposition 3.2}

Proof. We have by Lemma 2.1 that

$$
A\left[\alpha_{f}\right]=\frac{\kappa-1}{4 \pi} \frac{\operatorname{Vol} X_{0}(N)}{\zeta^{(N)}(2)} \sum_{f \in H_{\kappa}^{\star}(N, \epsilon)} \omega_{f} \alpha_{f} L^{(N)}\left(1, \operatorname{Ad}^{2} f\right)=\frac{\kappa-1}{4 \pi} \frac{\operatorname{Vol} X_{0}(N)}{\zeta^{(N)}(2)} A^{h}\left[\alpha_{f} L^{(N)}\left(1, \operatorname{Ad}^{2} f\right)\right] .
$$

Recall that we have set $\varrho_{f}(n)$ to be the Dirichlet series coefficients of $L^{(N)}\left(s, \mathrm{Ad}^{2} f\right)$, along with

$$
\omega_{f}(x)=\sum_{n \leqslant x} \frac{\varrho_{f}(n)}{n}, \text { and } \omega_{f}(x, y)=\sum_{x<n \leqslant y} \frac{\varrho_{f}(n)}{n} .
$$

Lemma 6.1. We have

$$
L^{(N)}\left(1, \operatorname{Ad}^{2} f\right)=\omega_{f}(x)+\omega_{f}(x, y)+O_{\varepsilon}\left((N \kappa)^{\frac{1}{2}} y^{-\frac{1}{2}+\varepsilon}\right) .
$$

Assuming the generalized Lindelof hypothesis, the $(N \kappa)^{1 / 2}$ can be reduced to $(N \kappa)^{\varepsilon}$.

Proof (sketch). For $c, T, y>0$, we apply Perron's formula (see e.g. [Dav00, pg. 105]) to calculate $\omega_{f}(y)$, finding

$$
\omega_{f}(y)=\frac{1}{2 \pi i} \int_{c-i T}^{c+i T} L^{(N)}\left(1+s, \operatorname{Ad}^{2} f\right) \frac{y^{s}}{s} d s+O\left(y^{c} \sum_{n \geqslant 1} \frac{\varrho_{f}(n)}{n^{1+c}} \min \left(1, T^{-1}|\log y / n|^{-1}\right)\right) .
$$


We shift the contour to $\operatorname{Re}(s)=-2$ to get

$$
\begin{aligned}
\omega_{f}(y)=L^{(N)}\left(1, \operatorname{Ad}^{2} f\right)+\frac{1}{2 \pi i}\left(\int_{c-i T}^{-2-i T}+\int_{-2-i T}^{-2+i T}\right. & \left.+\int_{-2+i T}^{c+i T}\right) L^{(N)}\left(1+s, \operatorname{Ad}^{2} f\right) \frac{y^{s}}{s} d s \\
& +O\left(y^{c} \sum_{n \geqslant 1} \frac{\varrho_{f}(n)}{n^{1+c}} \min \left(1, T^{-1}|\log y / n|^{-1}\right)\right) .
\end{aligned}
$$

By an inspection of the functional equation for $L(s, f \otimes \bar{f})$ found in [Li79, Example 1], we have the convexity bound (see e.g. [IK04, (5.20)])

$$
L^{(N)}\left(s, \operatorname{Ad}^{2} f\right) \ll\left[(\kappa N)^{2}(1+|t|)^{3}\right]^{\frac{1-\sigma}{2}+\varepsilon},
$$

where $s=\sigma+i t$, valid for $\sigma \leqslant 1$. Choosing $c=\varepsilon, T=(N \kappa)^{-\frac{1}{2}} y^{\frac{1}{2}+\varepsilon}$, and estimating all of the terms in (6.1) directly, one finds the estimate in the statement of the Lemma.

If one assumes the generalized Lindelöf hypothesis in place of (6.2), then we shift the contour to $\operatorname{Re}(s)=-1 / 2$ instead of -2 and follow the same steps.

By Lemma 6.1 we have

$$
A\left[\alpha_{f}\right]=\frac{\kappa-1}{4 \pi} \frac{\operatorname{Vol} X_{0}(N)}{\zeta^{(N)}(2)}\left(A^{h}\left[\omega_{f}(x) \alpha_{f}\right]+A^{h}\left[\omega_{f}(x, y) \alpha_{f}\right]+O\left((N \kappa)^{\frac{1}{2}} y^{-\frac{1}{2}+\varepsilon} A^{h}\left[\left|\alpha_{f}\right|\right]\right)\right) .
$$

By the hypothesis (3.3) we have $A^{h}\left[\left|\alpha_{f}\right|\right] \ll_{\varepsilon}(N \kappa)^{\varepsilon}$, and so taking $y=(N \kappa)^{3+\varepsilon}$, we find that the $O$ term in $(6.3)$ is $\ll(N \kappa)^{-1}$.

Next we consider the second term and treat it using the following large sieve inequality. This is a slight variation on Corollary 6 of [DK00], see also [KM99, Prop. 1]. Let $\lambda_{f}^{(2)}(n)$ be the Dirichlet series coefficients of the automorphic adjoint-square $L$-function $L\left(s, \mathrm{Ad}^{2} \pi\right)$, where $f$ is a newform for the representation $\pi$. If $(n, N)=1$ then we have that $\lambda_{f}^{(2)}(n)=\varrho_{f}(n)$.

Proposition 6.2. Let $X \geqslant(N \kappa)^{8}$. We have for all $\varepsilon>0$ that

$$
\sum_{f \in H_{\kappa}^{\star}(N, \epsilon)}\left|\sum_{n \leqslant X} a_{n} \lambda_{f}^{(2)}(n)\right|^{2} \ll_{\varepsilon} X^{1+\varepsilon} \sum_{n \leqslant X}\left|a_{n}\right|^{2}
$$

for any finite family $\left(a_{n}\right)_{1 \leqslant n \leqslant X}$ of complex numbers, where the constant depends only on $\varepsilon$.

By following closely Kowalski and Michel [KM99, §3.3] one deduces from Proposition [6.2 the following Lemma.

Lemma 6.3. Let $r \geqslant 1$ be an integer such that $x^{r} \geqslant(N \kappa)^{10}$. Then for all $\varepsilon>0$ we have

$$
A\left[\omega_{f}(x, y)^{2 r}\right] \ll_{r, \varepsilon}(N \kappa)^{\varepsilon},
$$

where the implied constant depends only on $r$ and $\varepsilon$.

Proof. It suffices to replace instances of $\lambda_{f}\left(n^{2}\right)$ in [KM99, Lemma 3] by $\bar{\epsilon}(n) \lambda_{f}\left(n^{2}\right)$ and to use equations (2.3) and (2.4) in the place of the equations (15) and (16) of Kowalski and Michel.

We now can give an estimate for the second term of (6.3). We use Hölder's inequality to separate $\omega_{f}(x, y)$ from $\alpha_{f}$, and Lemma 6.3 to handle the former. Precisely, let $s$ be defined by 
$(2 r)^{-1}+s^{-1}=1$. Applying Hölder's inequality we find for any integer $r \geqslant 1$ that

$$
\begin{aligned}
A^{h}\left[\omega_{f}(x, y) \alpha_{f}\right] & =\sum_{f \in H_{\kappa}^{\star}} \omega_{f} \omega_{f}(x, y) \alpha_{f} \\
& \leqslant A\left[\omega_{f}(x, y)^{2 r}\right]^{\frac{1}{2 r}}\left(\sum_{f \in H_{\kappa}^{\star}(N, \epsilon)}\left(\omega_{f}\left|\alpha_{f}\right|\right)^{s}\right)^{\frac{1}{s}} \\
& \leqslant A^{\frac{1}{2 r}} A\left[\omega_{f}(x, y)^{2 r}\right]^{\frac{1}{2 r}} A^{h}\left[\left|\alpha_{f}\right|\right]^{\frac{1}{s}},
\end{aligned}
$$

where

$$
A=\max _{f \in H_{\kappa}^{\star}(N, \epsilon)} \omega_{f}\left|\alpha_{f}\right| \ll_{\varepsilon}(N \kappa)^{-\delta+\varepsilon}
$$

by hypothesis (3.4). Suppose now that $r$ is sufficiently large so that $x^{r} \geqslant(N \kappa)^{10}$. Then Lemma 6.3 applies, and we have

$$
A\left[\omega_{f}(x, y)^{2 r}\right]^{\frac{1}{2 r}} \ll_{r, \varepsilon}(N \kappa)^{\varepsilon} .
$$

Lastly, by hypothesis (3.3) we have

$$
A^{h}\left[\left|\alpha_{f}\right|\right]^{\frac{1}{s}} \ll_{\varepsilon}(N \kappa)^{\varepsilon} .
$$

Putting these estimates together, we find that $A^{h}\left[\omega_{f}(x, y) \alpha_{f}\right] \ll_{r, \varepsilon}(N \kappa)^{-\frac{\delta}{2 r}+\varepsilon}$, and so derive the bound claimed in Proposition 3.2 .

\section{Proof of Theorem 3.1}

Proof. The strategy of the proof is the pick an orthogonal basis for $S_{\kappa}\left(\Gamma_{0}(N), \epsilon\right)$ and compute the Fourier coefficients of basis elements explicitly. For $f$ a modular function of weight $\kappa$, we denote by $f_{\mid d}(z)=d^{\frac{\kappa}{2}} f(d z)$. Atkin-Lehner theory gives an orthogonal direct sum decomposition

$$
S_{\kappa}\left(\Gamma_{0}(N), \epsilon\right)=\bigoplus_{L M=N} \bigoplus_{f \in H_{\kappa}^{\star}(M, \epsilon)} S_{\kappa}(L ; f, \epsilon),
$$

where $S_{\kappa}(L ; f, \epsilon)=\operatorname{span}\left\{f_{\mid \ell}: \ell \mid L\right\}$ is called an oldclass. Note that the inner sum is $\{0\}$ unless $\mathbf{c}(\epsilon) \mid M$, so we may assume this for the remainder of the proof.

To pick an orthogonal basis for $S_{\kappa}\left(\Gamma_{0}(N), \epsilon\right)$ it then suffices to pick a orthonormal basis for each oldclass $S_{\kappa}(L ; f, \epsilon)$. We use a basis for the oldclasses first due to Schulze-Pillot/Yenirce SPY18, Thm. 8]. The basis constructed by Schulze-Pillot/Yenirce is the same as the one found by Rouymi [Rou11] in the case of prime power level and trivial nebentypus and $\mathrm{Ng}$ Ng12 in the case of arbitrary level and trivial nebentypus, see also Blomer and Milićević [BM15, Ch. 5] and Humphries Hum18, Lemma 3.15]. Each of these preceding works used the Rankin-Selberg method to compute inner products and orthonomalize the oldclasses. Schulze-Pillot/Yenirce however took a different and simpler path, using the trace operator to compute the inner products. 
Let $f \in H^{\star}(M, \epsilon)$. For integers $d \mid g$ one defines a joint multiplicative function $\xi_{g}(d)$. On prime powers $\xi_{g}(d)$ is given for $\nu \geqslant 2$ as follows:

$$
\begin{aligned}
\xi_{1}(1) & =1, & \xi_{p^{\nu}}\left(p^{\nu}\right) & =\left(1-\frac{\left|\lambda_{f}(p)\right|^{2}}{p\left(1+\frac{\varepsilon_{0, M}(p)}{p}\right)^{2}}\right)^{-\frac{1}{2}}\left(1-\frac{\varepsilon_{0, M}(p)^{2}}{p^{2}}\right)^{-\frac{1}{2}}, \\
\xi_{p}(p) & =\left(1-\frac{\left|\lambda_{f}(p)\right|^{2}}{p\left(1+\frac{\epsilon_{0, M}(p)}{p}\right)^{2}}\right)^{-\frac{1}{2}}, & \xi_{p^{\nu}}\left(p^{\nu-1}\right) & =\frac{-\overline{\lambda_{f}(p)}}{\sqrt{p}} \xi_{p^{\nu}}\left(p^{\nu}\right), \\
\xi_{p}(1) & =\frac{-\overline{\lambda_{f}(p)}}{\sqrt{p}\left(1+\epsilon_{0, M}(p) / p\right)} \xi_{p}(p), & \xi_{p^{\nu}}\left(p^{\nu-2}\right) & =\frac{\overline{\epsilon(p)}}{p} \xi_{p^{\nu}}\left(p^{\nu}\right),
\end{aligned}
$$

and $\xi_{p^{a}}\left(p^{b}\right)=0$ in all other cases.

Proposition 7.1 (Thm. 9 [SPY18] $)$. Let $M \mid N$ and let $f \in H_{\kappa}^{\star}(M, \epsilon)$. The set of functions

$$
\left\{f^{(g)}(z)=\sum_{d \mid g} \xi_{g}(d) d^{\frac{\kappa}{2}} f(d z): g \mid L\right\}
$$

is an orthogonal basis for $S_{k}(L ; f, \epsilon)$. In fact, if $f$ is $L^{2}\left(\Gamma_{0}(N) \backslash \mathcal{H}\right)$-normalized, then the above set is in fact orthonormal.

Now that we have an orthonormal basis for $S_{\kappa}\left(\Gamma_{0}(N), \epsilon\right)$, we follow Barrett, Burkhardt, DeWitt, Dorward, and Miller [BBD ${ }^{+} 17$ ] to derive the Petersson formula for newforms Theorem 3.1 ,

Let $f \in H_{\kappa}^{\star}(M, \epsilon)$ have Fourier coefficients $a_{f}(n)$ and be normalized so that $a_{f}(1)=1$. Of course $f(z) /\|f\|_{N}$ is $L^{2}\left(\Gamma_{0}(N) \backslash \mathcal{H}\right)$-normalized, so using the basis in Proposition 7.1 we have

$$
\begin{aligned}
\Delta_{\kappa, N, \epsilon}(m, n) & =\frac{c_{\kappa}}{(m n)^{\frac{\kappa-1}{2}}} \sum_{g \in \mathcal{B}_{\kappa}\left(\Gamma_{0}(N), \epsilon\right)} b_{g}(n) \overline{b_{g}(m)} \\
& =\frac{c_{\kappa}}{(m n)^{\frac{\kappa-1}{2}}} \sum_{L M=N} \sum_{f \in H_{\kappa}^{\star}(m, \epsilon)} \frac{1}{\langle f, f\rangle_{N}} \sum_{g \mid L} \overline{a_{f^{(g)}(m)}} a_{f^{(g)}}(n) .
\end{aligned}
$$

By definition of $f^{(g)}$ we have

$$
a_{f^{(g)}}(n)=\sum_{d \mid(g, n)} \xi_{g}(d) d^{\frac{\kappa}{2}} a_{f}\left(\frac{n}{d}\right),
$$

which are now expressible in terms of Hecke eigenvalues $\lambda_{f}(n)$ normalized so that $\left|\lambda_{f}(n)\right| \leqslant d(n)$. We have then that

$$
\begin{aligned}
& \Delta_{\kappa, N, \epsilon}(m, n)=\frac{c_{\kappa}}{(m n)^{\frac{\kappa-1}{2}}} \sum_{L M=N} \sum_{f \in H_{\kappa}^{\star}(M, \epsilon)} \frac{1}{\|f\|_{N}^{2}} \sum_{g \mid L} \overline{\left(\sum_{d \mid(g, m)} \xi_{g}(d) d^{\frac{\kappa}{2}} a_{f}\left(\frac{m}{d}\right)\right)}\left(\sum_{d \mid(g, n)} \xi_{g}(d) d^{\frac{\kappa}{2}} a_{f}\left(\frac{n}{d}\right)\right) \\
& =c_{\kappa} \sum_{L M=N} \sum_{f \in H_{\kappa}^{\star}(M, \epsilon)} \frac{1}{\|f\|_{N}^{2}} \sum_{g \mid L} \overline{\left(\sum_{d \mid(g, m)} \xi_{g}(d) d^{\frac{1}{2}} \lambda_{f}\left(\frac{m}{d}\right)\right)}\left(\sum_{d \mid(g, n)} \xi_{g}(d) d^{\frac{1}{2}} \lambda_{f}\left(\frac{n}{d}\right)\right) \\
& =c_{\kappa} \sum_{L M=N} \sum_{f \in H_{\kappa}^{\star}(N, \epsilon)} \frac{1}{\|f\|_{N}^{2}} \sum_{g \mid L} \Xi_{g}(m, n, f),
\end{aligned}
$$

where we have set

$$
\Xi_{g}(m, n, f)=\overline{\left(\sum_{d \mid(g, m)} \xi_{g}(d) d^{\frac{1}{2}} \lambda_{f}\left(\frac{m}{d}\right)\right)}\left(\sum_{d \mid(g, n)} \xi_{g}(d) d^{\frac{1}{2}} \lambda_{f}\left(\frac{n}{d}\right)\right)
$$


for $g|L| N$.

Now suppose that $\left(d_{1}, d_{2}\right)=1$ and $d_{1} d_{2} \mid m$. Then by Hecke multiplicativity we have

$$
\lambda_{f}\left(\frac{m}{d_{1}}\right) \lambda_{f}\left(\frac{m}{d_{2}}\right)=\lambda_{f}(m) \lambda_{f}\left(\frac{m}{d_{1} d_{2}}\right),
$$

so that for $\left(g_{1}, g_{2}\right)=1$ we have

$$
\Xi_{g_{1}}(m, n, f) \Xi_{g_{2}}(m, n, f)=\overline{\lambda_{f}(m)} \lambda_{f}(n) \Xi_{g_{1} g_{2}}(m, n, f) .
$$

Therefore

$$
\Delta_{\kappa, N, \epsilon}(m, n)=c_{\kappa} \sum_{L M=N} \sum_{f \in H_{\kappa}^{\star}(M, \epsilon)} \frac{1}{\|f\|_{N}^{2}}\left(\overline{\lambda_{f}(m)} \lambda_{f}(n)\right)^{1-\omega(L)} \prod_{p^{\alpha} \| L}\left(\sum_{d \mid p^{\alpha}} \Xi_{d}(m, n, f)\right),
$$

where $\omega(n)$ is the number of distinct prime factors of $n$. Let

$$
V_{p^{\alpha}}(m, n, f)=\sum_{d \mid p^{\alpha}} \Xi_{d}(m, n, f)=(1 * \Xi)_{p^{\alpha}}(m, n, f),
$$

where $*$ denotes Dirichlet convolution. We suppose now that $(m, n, N)=1$ and calculate.

Lemma 7.2 $\left(\mathrm{BBD}^{+} 17\right]$ Appendix A). If $(m, n, N)=1$ then we have

$$
\begin{aligned}
& V_{p^{\alpha}}(m, n, f)=\overline{\lambda_{f}(m)} \lambda_{f}(n)\left(1+\left|\xi_{p}(1)\right|^{2}+\left|\xi_{p^{2}}(1)\right|^{2}\right) \\
& +\delta_{p \mid m} \overline{\lambda_{f}(m / p)} \lambda_{f}(n) p^{\frac{1}{2}}\left(\overline{\xi_{p}(p)} \xi_{p}(1)+\overline{\xi_{p^{2}}(p)} \xi_{p^{2}}(1)\right) \\
& +\delta_{p \mid n} \overline{\lambda_{f}(m)} \lambda_{f}(n / p) p^{\frac{1}{2}}\left(\overline{\xi_{p}(1)} \xi_{p}(p)+\overline{\xi_{p^{2}}(1)} \xi_{p^{2}}(p)\right) \\
& +\delta_{p^{2} \mid m} \overline{\lambda_{f}\left(m / p^{2}\right)} \lambda_{f}(n) p \overline{\xi_{p^{2}}\left(p^{2}\right)} \xi_{p^{2}}(1)+\delta_{p^{2} \mid n} \overline{\lambda_{f}(m)} \lambda_{f}\left(n / p^{2}\right) p \overline{\xi_{p^{2}}(1)} \xi_{p^{2}}\left(p^{2}\right),
\end{aligned}
$$

if $\alpha \geqslant 2$ and

$$
\begin{aligned}
V_{p^{\alpha}}(m, n, f)= & \overline{\lambda_{f}(m)} \lambda_{f}(n)\left(1+\left|\xi_{p}(1)\right|^{2}\right) \\
& +\delta_{p \mid m} \overline{\lambda_{f}(m / p)} \lambda_{f}(n) p^{\frac{1}{2}} \overline{\xi_{p}(p)} \xi_{p}(1) \\
& +\delta_{p \mid n} \overline{\lambda_{f}(m)} \lambda_{f}(n / p) p^{\frac{1}{2}} \overline{\xi_{p}(1)} \xi_{p}(p),
\end{aligned}
$$

if $\alpha=1$.

Proof. We actually have if $\alpha \geqslant 2$ that

$$
V_{p^{\alpha}}(m, n, f)=\Xi_{1}(m, n, f)+\Xi_{p}(m, n, f)+\Xi_{p^{2}}(m, n, f) .
$$

The other summands vanish because by our assumption $(m, n, N)=1$, since if $p \mid m$ then $p \nmid n$ because $p \mid N$. So each $p$ divides either $m$ or $n$ but never both. Then, we have that $\xi_{p^{\beta}}(1)=0$ for $\beta \geqslant 3$. In fact, even more terms vanish. We have

$$
\begin{aligned}
& V_{p^{\alpha}}(m, n, f)=\overline{\lambda_{f}(m)} \lambda_{f}(n)\left(1+\left|\xi_{p}(1)\right|^{2}+\left|\xi_{p^{2}}(1)\right|^{2}\right) \\
& +\delta_{p \mid m} \overline{\lambda_{f}(m / p)} \lambda_{f}(n) p^{\frac{1}{2}}\left(\overline{\xi_{p}(p)} \xi_{p}(1)+\overline{\xi_{p^{2}}(p)} \xi_{p^{2}}(1)\right) \\
& +\delta_{p \mid n} \overline{\lambda_{f}(m)} \lambda_{f}(n / p) p^{\frac{1}{2}}\left(\overline{\xi_{p}(1)} \xi_{p}(p)+\overline{\xi_{p^{2}}(1)} \xi_{p^{2}}(p)\right) \\
& +\delta_{p^{2} \mid m} \overline{\lambda_{f}\left(m / p^{2}\right)} \lambda_{f}(n) p \overline{\xi_{p^{2}}\left(p^{2}\right)} \xi_{p^{2}}(1)+\delta_{p^{2} \mid n} \overline{\lambda_{f}(m)} \lambda_{f}\left(n / p^{2}\right) p \overline{\xi_{p^{2}}(1)} \xi_{p^{2}}\left(p^{2}\right) .
\end{aligned}
$$

Inserting the formulas for $\xi$, we complete the proof. The formula for the $\alpha=1$ case is even simpler as we can drop the $p^{2}$ terms.

Recall we write $M L=N$ and $f \in H_{\kappa}^{\star}(M, \epsilon)$. 
Lemma 7.3. If $(m, N)=1$ and $(n, N)=1$ then we have

$$
\left(\overline{\lambda_{f}(m)} \lambda_{f}(n)\right)^{1-\omega(L)} \prod_{p^{\alpha}|| L} V_{p^{\alpha}}(m, n, f)=\overline{\lambda_{f}(m)} \lambda_{f}(n) \prod_{p|| L}\left(1+\left|\xi_{p}(1)\right|^{2}\right) \prod_{p^{2} \mid L}\left(1+\left|\xi_{p}(1)\right|^{2}+\left|\xi_{p^{2}}(1)\right|^{2}\right) .
$$

Proof. Note that the conditions $(m, N)=1$ and $(n, N)=1$ imply that $p \nmid m$ and $p \nmid n$. So the formula above follows immediately from the formulas in Lemma 7.2 .

One has that $\|f\|_{N}^{2}=\frac{\psi(N)}{\psi(M)}\|f\|_{M}^{2}$ since $f \in H_{\kappa}^{\star}(M, \epsilon)$. Thus

$$
\begin{aligned}
\Delta_{\kappa, N, \epsilon}(m, n)=c_{\kappa} \sum_{L M=N} \frac{\psi(M)}{\psi(N)} \sum_{f \in H_{\kappa}^{\star}(M, \epsilon)} \frac{1}{\|f\|_{M}^{2}} \overline{\lambda_{f}(m)} \lambda_{f}(n) \prod_{p \| L}\left(1+\left|\xi_{p}(1)\right|^{2}\right) \\
\times \prod_{p^{2} \mid L}\left(1+\left|\xi_{p}(1)\right|^{2}+\left|\xi_{p^{2}}(1)\right|^{2}\right) .
\end{aligned}
$$

Next we insert the definitions of the $\xi$ functions. Let

$$
r_{f}(p)=1-\frac{\left|\lambda_{f}(p)\right|^{2}}{p\left(1+\frac{\epsilon_{0, M}(p)}{p}\right)^{2}}
$$

so

$$
r_{f}(p)^{-1}=1+\frac{\left|\lambda_{f}(p)\right|^{2}}{p\left(1+\frac{\epsilon_{0, M}(p)}{p}\right)^{2}}+\left(\frac{\left|\lambda_{f}(p)\right|^{2}}{p\left(1+\frac{\epsilon_{0, M}(p)}{p}\right)^{2}}\right)^{2}+\cdots,
$$

where $\epsilon_{0, M}$ denotes the trivial character modulo $M$. Observe that

$$
1+\left|\xi_{p}(1)\right|^{2}=r_{f}(p)^{-1}
$$

and

Then we get

$$
1+\left|\xi_{p}(1)\right|^{2}+\left|\xi_{p^{2}}(1)\right|^{2}=r_{f}(p)^{-1}\left(1-\frac{\epsilon_{0, M}(p)}{p^{2}}\right)^{-1}
$$

$$
\Delta_{\kappa, N, \epsilon}(m, n)=c_{\kappa} \sum_{L M=N} \frac{\psi(M)}{\psi(N)} \prod_{p^{2} \mid L}\left(1-\frac{\epsilon_{0, M}(p)}{p^{2}}\right)^{-1} \sum_{f \in H_{\kappa}^{\star}(M, \epsilon)} \frac{\overline{\lambda_{f}(m)} \lambda_{f}(n)}{\|f\|_{M}^{2}} \prod_{p \mid L} \frac{1}{r_{f}(p)} .
$$

Next we need a formula for $r_{f}(p)^{-1}$. Recall from (2.1) that at a prime $p \nmid M$ that the local adjoint square $L$ function is given by

$$
L_{p}\left(1, \operatorname{Ad}^{2} f\right)=\frac{1}{1-p^{-2}} \sum_{\alpha \geqslant 0} \frac{\bar{\epsilon}\left(p^{\alpha}\right) \lambda_{f}\left(p^{2 \alpha}\right)}{p^{\alpha}}=\frac{1}{\left(1-\frac{\alpha(p) / \beta(p)}{p}\right)\left(1-\frac{1}{p}\right)\left(1-\frac{\beta(p) / \alpha(p)}{p}\right)}
$$

so that

$$
\begin{aligned}
\sum_{\alpha \geqslant 0} \frac{\bar{\epsilon}\left(p^{\alpha}\right) \lambda_{f}\left(p^{2 \alpha}\right)}{p^{\alpha}} & =\frac{1+\frac{1}{p}}{\left(1-\frac{\alpha(p) / \beta(p)}{p}\right)\left(1-\frac{\beta(p) / \alpha(p)}{p}\right)} \\
& =\frac{1+\frac{1}{p}}{\left(1+\frac{1}{p}\right)^{2}-\frac{\left|\lambda_{f}(p)\right|^{2}}{p}} \\
& =\frac{1}{\left(1+\frac{1}{p}\right) r_{f}(p)} \\
20 &
\end{aligned}
$$


where the second equals sign follows from the formulas

$$
\left|\lambda_{f}(p)\right|^{2}=\bar{\epsilon}(p) \lambda_{f}(p)^{2}, \quad \lambda_{f}(p)=\alpha(p)+\beta(p), \quad \alpha(p) \beta(p)=\epsilon(p)
$$

which are valid when $p \nmid M$. We can summarize the above calculation and Lemma 2.2 as:

$$
r_{f}(p)^{-1}= \begin{cases}\left(1+\frac{1}{p}\right) \sum_{\alpha \geqslant 0} \frac{\bar{\epsilon}\left(p^{\alpha}\right) \lambda_{f}\left(p^{2 \alpha}\right)}{p^{\alpha}} & \text { if } p \nmid M \\ \left(1-\frac{a_{M, \epsilon}(p)}{p}\right)^{-1} & \text { if } p \mid M .\end{cases}
$$

Let

$$
\Delta_{\kappa, N, \epsilon}^{\star}(m, n)=c_{\kappa} \sum_{f \in H_{\kappa}^{\star}(N, \epsilon)} \frac{\overline{\lambda_{f}(m)} \lambda_{f}(n)}{\|f\|_{N}^{2}} .
$$

Recall the definition of $R(M, L, \epsilon)$ from the statement of Theorem 3.1, which we rearrange to

$$
R(M, L, \epsilon)=\frac{\psi(M)}{\psi(M L)} \prod_{\substack{p^{2} \mid L \\ p \nmid M}}\left(1-\frac{1}{p^{2}}\right)^{-1} \prod_{\substack{p \mid L \\ p \nmid M}}\left(1+\frac{1}{p}\right) \prod_{p \mid(M, L)}\left(1-\frac{a_{M, \epsilon}(p)}{p}\right)^{-1}
$$

We have then that

$$
\Delta_{\kappa, N, \epsilon}(m, n)=\sum_{L M=N} R(M, L, \epsilon) \sum_{\substack{\ell \mid L^{\infty} \\(\ell, M)=1}} \frac{\bar{\epsilon}(\ell)}{\ell} \Delta_{\kappa, M, \epsilon}^{\star}\left(m, n \ell^{2}\right) .
$$

This is analogous to the first half of $\left[\mathrm{BBD}^{+} 17\right.$, Prop. 4.1]. Now we would like to invert this formula, and we prepare for this with two lemmas.

Lemma 7.4. Let $\alpha, \beta \geqslant 0$ and $0 \leqslant \gamma \leqslant \beta$ and $\mathbf{c}_{p}(\epsilon) \leqslant \beta-1$. Then

$$
R\left(p^{\beta}, p^{\alpha}, \epsilon\right) R\left(p^{\gamma}, p^{\beta-\gamma}, \epsilon\right)=R\left(p^{\gamma}, p^{\alpha+\beta-\gamma}, \epsilon\right) .
$$

Proof. We check cases.

Case $\alpha \geqslant 0$ and $\beta=\gamma$. Note that $R\left(p^{\gamma}, 1, \epsilon\right)=1$ for any $\gamma \geqslant 0$.

Case $\alpha=0$. Note that $R\left(p^{\beta}, 1, \epsilon\right)=1$ for any $\beta \geqslant 0$.

Case $\alpha \geqslant 1, \beta=1$ and $\gamma=0$. We have by hypothesis $\mathbf{c}_{p}(\epsilon)=0$, so

$$
R\left(p, p^{\alpha}\right) R(1, p)=\frac{\psi(p)}{\psi\left(p^{\alpha+1}\right)}\left(1-\frac{1}{p^{2}}\right)^{-1} \frac{1}{\psi(p)}\left(1+\frac{1}{p}\right) .
$$

On the other hand, we also have

$$
R\left(1, p^{\alpha+1}\right)=\frac{1}{\psi\left(p^{\alpha+1}\right)}\left(1-\frac{1}{p^{2}}\right)^{-1}\left(1+\frac{1}{p}\right) .
$$

Case $\alpha \geqslant 1, \beta \geqslant 2$ and $\gamma=0$. We have $p \mid\left(p^{\beta}, p^{\alpha}\right)$ and $a_{p^{\beta}, \epsilon}(p)=0$, so $R\left(p^{\beta}, p^{\alpha}, \epsilon\right)=p^{-\alpha}$ and

$$
R\left(1, p^{\beta}, \epsilon\right)=\frac{1}{\psi\left(p^{\beta}\right)}\left(1-\frac{1}{p^{2}}\right)^{-1}\left(1+\frac{1}{p}\right),
$$

and

$$
R\left(1, p^{\alpha+\beta}, \epsilon\right)=\frac{1}{\psi\left(p^{\alpha+\beta}\right)}\left(1-\frac{1}{p^{2}}\right)^{-1}\left(1+\frac{1}{p}\right) .
$$

Generic case $\alpha \geqslant 1, \beta \geqslant 2,1 \leqslant \gamma \leqslant \beta-1$, and $\mathbf{c}_{p}(\epsilon) \leqslant \beta-1$. We have

$$
R\left(p^{\beta}, p^{\alpha}, \epsilon\right)=\frac{\psi\left(p^{\beta}\right)}{\psi\left(p^{\alpha+\beta}\right)}
$$




$$
R\left(p^{\gamma}, p^{\beta-\alpha}, \epsilon\right)=\frac{\psi\left(p^{\gamma}\right)}{\psi\left(p^{\beta}\right)}\left(1-\frac{a_{p^{\gamma}, \epsilon}(p)}{p}\right)^{-1}
$$

and

$$
R\left(p^{\gamma}, p^{\beta+\alpha-\gamma}, \epsilon\right)=\frac{\psi\left(p^{\gamma}\right)}{\psi\left(p^{\alpha+\beta}\right)}\left(1-\frac{a_{p^{\gamma}, \epsilon}(p)}{p}\right)^{-1} .
$$

The above cover all the cases in the lemma.

Lemma 7.5. Let $N \in \mathbf{N}, N=L M$, and $M=W Q$. Then

$$
R(M, L, \epsilon) R(W, Q, \epsilon) \delta_{\mathbf{c}(\epsilon) \mid W}=R(W, L Q, \epsilon) \delta_{\mathbf{c}(\epsilon) \mid W} .
$$

Proof. Both sides of the desired formula are multiplicative. Let $\alpha=v_{p}(L), \beta=v_{p}(M)$, and $\gamma=v_{p}(W)$. It then suffices to check that

$$
R\left(p^{\beta}, p^{\alpha}, \epsilon\right) R\left(p^{\gamma}, p^{\beta-\gamma}, \epsilon\right) \delta_{\gamma \geqslant \mathbf{c}_{p}(\epsilon)}=R\left(p^{\gamma}, p^{\alpha+\beta-\gamma}, \epsilon\right) \delta_{\gamma \geqslant \mathbf{c}_{p}(\epsilon)} .
$$

If $\mathbf{c}_{p}(\epsilon) \leqslant \beta-1$ then (7.4) is true by Lemma 7.4. So, suppose not. Then $\beta \leqslant \mathbf{c}_{p}(\epsilon) \leqslant \gamma$, but $W \mid M$ so $\gamma \leqslant \beta$ and so $\beta=\gamma$. In the case $\beta=\gamma$ the equation (7.4) is true because $R\left(p^{\beta}, 1, \epsilon\right)=1$.

We are now prepared to invert (7.2) using Lemma 7.5, We calculate

$$
\begin{aligned}
& \sum_{L M=N} \mu(L) R(M, L, \epsilon) \sum_{\substack{\ell \mid L^{\infty} \\
(\ell, M)=1}} \frac{\bar{\epsilon}(\ell)}{\ell} \Delta_{\kappa, M, \epsilon}\left(m, n \ell^{2}\right) \\
= & \sum_{L M=N} \mu(L) R(M, L, \epsilon) \sum_{\substack{\ell \mid L^{\infty} \\
(\ell, M)=1}} \frac{\bar{\epsilon}(\ell)}{\ell} \sum_{Q W=M} R(W, Q, \epsilon) \sum_{\substack{q \mid Q^{\infty} \\
(q, W)=1}} \frac{\bar{\epsilon}(q)}{q} \Delta_{\kappa, W, \epsilon}^{\star}\left(m, n \ell^{2} q^{2}\right) \\
= & \sum_{L M=N} \mu(L) \sum_{Q W=M} R(M, L, \epsilon) R(W, Q, \epsilon) \sum_{\substack{\mid(L Q) \\
(b, W)=1}} \frac{\bar{\epsilon}(b)}{b} \Delta_{\kappa, W, \epsilon}^{\star}\left(m, n b^{2}\right) \\
= & \sum_{W X=N} R(W, X, \epsilon) \sum_{b \mid X^{\infty}} \frac{\bar{\epsilon}(b)}{b} \Delta_{\kappa, W, \epsilon}^{\star}\left(m, n b^{2}\right) \sum_{L Q=X} \mu(L) \\
= & R(N, 1, \epsilon) \Delta_{\kappa, N, \epsilon}^{\star}(m, n) \\
= & \Delta_{\kappa, N, \epsilon}^{\star}(m, n) .
\end{aligned}
$$

where the first equals sign is (17.2), the third is by Lemma 7.5, and the fourth is Mobius inversion.

\section{REFERENCES}

[AL70] A. O. L. Atkin and J. Lehner. Hecke operators on $\Gamma_{0}(m)$. Math. Ann., 185:134-160, 1970.

[Ban97] William D. Banks. Twisted symmetric-square $L$-functions and the nonexistence of Siegel zeros on GL(3). Duke Math. J., 87(2):343-353, 1997.

$\left[\mathrm{BBD}^{+} 17\right]$ O. Barrett, P. Burkhardt, J. DeWitt, R. Dorward, and S. J. Miller. One-level density for holomorphic cusp forms of arbitrary level. Res. Number Theory, 3, Art. 25, 21 pp., 2017.

[BBM17] Valentin Blomer, Jack Buttcane, and Péter Maga. Applications of the Kuznetsov formula on GL(3) II: the level aspect. Math. Ann., 369(1-2):723-759, 2017.

[BM15] Valentin Blomer and Djordje Milićević. The second moment of twisted modular L-functions. Geom. Funct. Anal., 25(2):453-516, 2015.

[CDF97] J. B. Conrey, W. Duke, and D. W. Farmer. The distribution of the eigenvalues of Hecke operators. Acta Arith., 78(4):405-409, 1997.

[Dav00] Harold Davenport. Multiplicative number theory, volume 74 of Graduate Texts in Mathematics. SpringerVerlag, New York, third edition, 2000. Revised and with a preface by Hugh L. Montgomery. 
[DI95] Fred Diamond and John Im. Modular forms and modular curves. In Seminar on Fermat's Last Theorem (Toronto, ON, 1993-1994), volume 17 of CMS Conf. Proc., pages 39-133. Amer. Math. Soc., Providence, RI, 1995.

[DK00] W. Duke and E. Kowalski. A problem of Linnik for elliptic curves and mean-value estimates for automorphic representations. Invent. Math., 139(1):1-39, 2000. With an appendix by Dinakar Ramakrishnan.

[DS05] Fred Diamond and Jerry Shurman. A first course in modular forms, volume 228 of Graduate Texts in Mathematics. Springer-Verlag, New York, 2005.

[GHL94] Dorian Goldfeld, Jeffery Hoffstein, and Daniel Lieman. Appendix: An effective zero-free region. Ann. of Math. (2), 140(2):177-181, 1994.

[GJ78] Stephen Gelbart and Hervé Jacquet. A relation between automorphic representations of GL(2) and GL(3). Ann. Sci. École Norm. Sup. (4), 11(4):471-542, 1978.

[HB81] D. R. Heath-Brown. The fourth power mean of Dirichlet's L-functions. Analysis, 1(1):25-32, 1981.

[Hum18] P. Humphries. Density theorems for exceptional eigenvalues for congruence subgroups. Algebra Number Theory, to appear, 2018.

[Igu59] Jun-ichi Igusa. Kroneckerian model of fields of elliptic modular functions. Amer. J. Math., 81:561-577, 1959.

[IK04] Henryk Iwaniec and Emmanuel Kowalski. Analytic number theory, volume 53 of American Mathematical Society Colloquium Publications. American Mathematical Society, Providence, RI, 2004.

[ILS00] Henryk Iwaniec, Wenzhi Luo, and Peter Sarnak. Low lying zeros of families of L-functions. Inst. Hautes Études Sci. Publ. Math., (91):55-131 (2001), 2000.

[Iwa97] Henryk Iwaniec. Topics in classical automorphic forms, volume 17 of Graduate Studies in Mathematics. American Mathematical Society, Providence, RI, 1997.

[KL13] A. Knightly and C. Li. Kuznetsov's trace formula and the Hecke eigenvalues of Maass forms. Mem. Amer. Math. Soc., 224(1055):vi+132, 2013.

[KM99] E. Kowalski and P. Michel. The analytic rank of $J_{0}(q)$ and zeros of automorphic $L$-functions. Duke Math. J., 100(3):503-542, 1999.

[KP17] Nathan Kaplan and Ian Petrow. Elliptic curves over a finite field and the trace formula. Proceedings of the London Mathematical Society, Published Online 2017.

[Li75] Wen Ch'ing Winnie Li. Newforms and functional equations. Math. Ann., 212:285-315, 1975.

[Li79] Wen Ch'ing Winnie Li. L-series of Rankin type and their functional equations. Math. Ann., 244(2):135166, 1979.

[Mil17] James S. Milne. Modular functions and modular forms (v1.31), 2017. Available at www.jmilne.org/math/.

[Mor91] Carlos Moreno. Algebraic curves over finite fields, volume 97 of Cambridge Tracts in Mathematics. Cambridge University Press, Cambridge, 1991.

[MS09] M. Ram Murty and Kaneenika Sinha. Effective equidistribution of eigenvalues of Hecke operators. J. Number Theory, 129(3):681-714, 2009.

[Mur95] M. Ram Murty. The analytic rank of $J_{0}(N)(\mathbf{Q})$. In Number theory (Halifax, NS, 1994), volume 15 of $C M S$ Conf. Proc., pages 263-277. Amer. Math. Soc., Providence, RI, 1995.

[Nel17] Paul D. Nelson. Analytic isolation of newforms of given level. Arch. Math. (Basel), 108(6):555-568, 2017.

[Ng12] Ming-ho Ng. The basis for space of cusp forms and Petersson trace formula. Masters thesis, University of Hong Kong, 2012.

[Ogg69] Andrew P. Ogg. On the eigenvalues of Hecke operators. Math. Ann., 179:101-108, 1969.

[PY18] Ian Petrow and Matthew P. Young. A generalized cubic moment and the Petersson formula for newforms. Math. Ann., published online, 2018.

[Ros92] Shepley L. Ross, II. A simplified trace formula for Hecke operators for $\Gamma_{0}(N)$. Trans. Amer. Math. Soc., 331(1):425-447, 1992.

[Rou11] D. Rouymi. Formules de trace et non-annulation de fonctions $L$ automorphes au niveau $\mathfrak{p}^{\nu}$. Acta Arith., 147(1):1-32, 2011.

[Ser97] Jean-Pierre Serre. Répartition asymptotique des valeurs propres de l'opérateur de Hecke $T_{p}$. J. Amer. Math. Soc., 10(1):75-102, 1997.

[SPY18] R. Schulze-Pillot and A. Yenirce. Petersson products of bases of spaces of cusp forms and estimates for Fourier coefficients. Int. J. Number Theory, 14(8):2277-2290, 2018.

[TVtZ82] M. A. Tsfasman, S. G. Vlăduț, and Th. Zink. Modular curves, Shimura curves, and Goppa codes, better than Varshamov-Gilbert bound. Math. Nachr., 109:21-28, 1982.

[Ven06] Akshay Venkatesh. Large sieve inequalities for GL(n)-forms in the conductor aspect. Adv. Math., 200(2):336-356, 2006.

[You17] Matthew P. Young. Explicit calculations with Eisenstein series. arXiv:1710.03624, 2017. 
ETH ZÜrich - Departement Mathematik, HG G 66.4, RÄmistrasse 101, 8092 Zürich, Switzerland E-mail address: ian.petrow@math.ethz.ch 